\title{
The chemical composition of the accretion disk and donor star in Ultra Compact X-ray Binaries: A comprehensive X-ray analysis
}

\author{
Filippos Koliopanos ${ }^{1,2 \star}$, Mathias Péault ${ }^{1,2}$, Georgios Vasilopoulos ${ }^{3}$ and Natalie Webb ${ }^{1,2}$. \\ ${ }^{1}$ CNRS, IRAP, 9 Av. colonel Roche, BP 44346, F-31028 Toulouse cedex 4, France \\ ${ }^{2}$ Université de Toulouse; UPS-OMP; IRAP, Toulouse, France \\ ${ }^{3}$ Department of Astronomy, Yale University, PO Box 208101, New Haven, CT 06520-8101, USA
}

Accepted XXX. Received YYY; in original form ZZZ

\begin{abstract}
We have analyzed the X-ray spectra of all known Ultra Compact X-ray Binaries (UCXBs), with the purpose of constraining the chemical composition of their accretion disk and donor star. Our investigation was focused on the presence (or absence) of the $\mathrm{Fe} \mathrm{K} \alpha$ emission line, which was used as the probe of chemical composition of the disk, based on previously established theoretical predictions for the reflection of X-ray radiation off the surface of C/O-rich or He-rich accretion disks in UCXBs. We have contrasted the results of our spectral analysis to the history of type I X-ray bursts from these systems, which can also indicate donor star composition. We found that UCXBs with prominent and persistent iron $\mathrm{K} \alpha$ emission also featured repeat bursting activity. On the other hand, the UCXBs for which no iron line was detected, appear to have few or no type I X-ray bursts detected over more than a decade of monitoring. Based on Monte Carlo simulations, demonstrating a strong correlation between the $\mathrm{Fe} \mathrm{K} \alpha$ line strength and the abundance of $\mathrm{C}$ and $\mathrm{O}$ in the accretion disk material and given the expected correlation between the $\mathrm{H} / \mathrm{He}$ abundance and the recurrence rate of type I $\mathrm{X}$-ray bursts, we propose that there is a considerable likelihood that UCXBs with persistent iron emission have He-rich donors, while those that do not, likely have $\mathrm{C} / \mathrm{O}$ or $\mathrm{O} / \mathrm{Ne} / \mathrm{Mg}$-rich donors. Our result strongly advocate for the development of more sophisticated simulations of X-ray reflection from hydrogen-poor accretion disks.
\end{abstract}

Key words: accretion, accretion discs - line: formation - line: profiles - X-rays: binaries

\section{INTRODUCTION}

Ultracompact X-ray binaries (UCXBs) are X-ray binary systems (XRBs) defined by their extremely short orbital periods of less than $\sim$ one hour. The orbital size defined by such short periods is too small to encompass the main sequence or red giant stars that make up for the donor star in typical low-mass XRBs (LMXBS), (e.g. Rappaport \& Joss 1984; Nelson, Rappaport \& Joss 1986b). UCXBs consist of a neutron star (although black holes have not been ruled out), that is accreting matter from degenerate companion star, a Roche-lobe filling white dwarf or helium star (e.g. Tutukov \& Yungelson 1993; Iben, Tutukov \& Yungelson 1995; Verbunt \& van den Heuvel 1995; Deloye \& Bildsten 2003; Deloye, Bildsten \& Nelemans 2005).

UCXB formation history can be divided into three major schemes. White dwarf (WD) neutron star (NS) binaries that gradually lose angular momentum due to gravitational radiation until the WD fills its Roche lobe and starts losing mass to the NS Pringle \& Webbink (1975). Semi-attached binaries reaching an exceptionally

^ E-mail: fkoliopanos@irap.omp.eu short orbital periods, resulting in high mass accretion rates from hydrogen-depleted non-degenerate donors Nelson et al. (1986a) or - in case of a companion star with a helium burning core at the time of contact - a helium star donor Savonije et al. (1986). Therefore, depending on the evolutionary track that leads to their creation UCXBs can have a variety of different donors ranging from Helium stars to $\mathrm{C} / \mathrm{O}$ or $\mathrm{O} / \mathrm{Ne} / \mathrm{Mg}$ WDs (e.g. Savonije, de Kool \& van den Heuvel 1986; Podsiadlowski, Rappaport \& Pfahl 2002; Yungelson, Nelemans \& van den Heuvel 2002; Bildsten \& Deloye 2004).

UCXBs offer the unique opportunity to study accretion of matter with extraordinary chemical composition. Furthermore, they are are unique laboratories for the study of binary evolution theory and particularly the common-envelope phase and its relevance to type Ia supernovae and are strong gravitational wave sources in the lowfrequency regime where the ESA/NASA LISA will be sensitive (e.g., Nelemans \& Jonker 2010; Amaro-Seoane et al. 2017; Cornish \& Robson 2017). They stand as excellent candidates for indepth multi-messenger studies of accretion onto compact sources. As population studies and disk stability and evolution analyses predict specific ratios of He-rich vs $\mathrm{C} / \mathrm{O}-\mathrm{O} / \mathrm{Ne} / \mathrm{Mg}$ systems, a key step in understanding of UCXB formation and evolution is the de- 


\section{Koliopanos et al.}

termination of the nature of the donor star composition and whether it lies in the one or the other category (Belczynski \& Taam 2004; Nelemans et al. 2010; Heinke et al. 2013).

The most straight forward approach to the determination of donor star composition would the spectral analysis of optical emission originating in the accretion disk and donor star. Indeed, for a fraction of UCXBs indications of extra-solar elemental abundances have been presented using optical and UV spectroscopy. Nelemans et al. (2004); Werner et al. (2006) and Nelemans et al. (2006) detected strong $\mathrm{C}$ and $\mathrm{O}$ lines in the optical spectra of two UCXBs (4U 1626-67, 4U 0614+09) which also lacked any evidence for the presence of $\mathrm{H}$ or $\mathrm{He}$, suggesting the two source may host $\mathrm{C} / \mathrm{O}$ rich donors. Furthermore, Nelemans et al. (2004) argued that 4U 1543$624 \& 2$ S 0918-549 show optical spectra similar to 4U 0614+09. On the other hand, a He/ $\mathrm{N}$ ratio consistent with a He-rich donor was indicated for 4 U 1916-05 (Nelemans et al. 2006). While optical spectroscopy can provide good indications for the chemical composition of UCXB donor stars, due to their small sized accretion disks (van Paradijs \& McClintock 1994) the optical emission from UCXBs is quite faint, with V-band absolute magnitudes typically larger than $\approx 5$ and for distances ranging from $\approx 3-12 \mathrm{kpc}$ (e.g. Nelemans et al. 2004, 2006). Therefore, establishing definitive evidence of the donor star composition - using optical spectroscopy - is a challenging task that can only be attempted using the latest generation of $>8 \mathrm{~m}$ telescopes.

As their name implies UCXBs are powerful sources of X-ray emission, the result of mass accretion at high mass accretion rates due to Roche lobe overflow of the donor star (see review by Nelemans \& Jonker 2010). Furthermore, the rate and stability of mass accretion depends strongly on the donor mass and chemical composition and therefore on the evolutionary track that for UCXB formation (e.g. Heinke et al. 2013, and references therein). This paper is focused on the X-ray emission of UCXBs and demonstrates how $\mathrm{X}$-ray spectroscopy, combined with numerical simulations of fundamental radiative processes, in the accretion disk and its vicinity can provide credible indications on the chemical composition of the accretion disks and donor stars of UCXB systems. In Section 2 we lay out the main principles of X-ray diagnostics of the chemical composition of UCXBs, followed by Section 3, where we present the results of X-ray spectroscopic analysis of all known UCBXs. We discuss our findings and present our conclusions in Sections 4 and 5 .

\section{X-RAY DIAGNOSTICS OF UCXBS}

The primary X-ray emission originates in the inner accretion disk and/or the surface layer hot optically thick matter on the NS, known as the boundary layer (Popham \& Sunyaev 2001). A secondary emission - evident by the hard X-ray emission with a non-thermal spectrum - originates in a corona of hot thermal electrons in the vicinity of the disk and central engine. The hot electrons up-scatter seed photons from the disk and boundary layer, producing emission with a spectrum characteristic of unsaturated thermal comptonization (e.g. Gilfanov 2010, and references therein). A fraction of these hard photons may be "reflected" (partially absorbed and re-emitted and/or scattered) off the accretion disk surface producing an additional emission component known as X-ray reflection (Basko et al. 1974; White et al. 1988; Lightman \& White 1988; George \& Fabian 1991).

X-ray reflection spectra are enriched with multiple emission lines - the result of K-shell emission from heavy elements present in the accreting material. However, most of the line photons are heavily diluted by the primary continuum photons (registered together with the reflected emission). Due to the high fluorescence yield and chemical abundance of iron, a bright $\mathrm{Fe} \mathrm{K} \alpha$ emission line at $\approx 6.4-6.9 \mathrm{keV}$ with an equivalent width (EW) typically of the order of $\approx 100 \mathrm{eV}$ (e.g. Cackett et al. 2010) is usually detected in the spectra of normal LMXBs with main sequence or red giant donors. Spectral analysis of the reflection component - and particularly $\mathrm{Fe}$ $\mathrm{K} \alpha$ line - can provide indications for the disk chemical composition and its physical parameters such as temperature and rotational velocity and size (e.g. Fabian et al. 1989; Matt et al. 1993, 1997; Ballantyne et al. 2001; García \& Kallman 2010; García et al. 2013; Koliopanos et al. 2013).

In the case of UCXBs, X-ray reflection can - in principle provide valuable insight into the abundances of chemical elements in the disk and donor star, thus revealing its nature. More specifically, the presence of oxygen and neon emission features - that appear in the spectra of reprocessed emission from the accretion disk and white dwarf surface - (e.g. Madej et al. 2010) and Kedges stemming from absorbing material in the vicinity of the disk (e.g. Schulz et al. 2010) could provide direct indication of a $\mathrm{C} / \mathrm{O}$ or $\mathrm{O} / \mathrm{Ne}$-rich disk and donor star. However, due to increased interstellar absorption below $1 \mathrm{keV}$ and contamination of the reflected component by the primary emission, detection of these features with sufficient accuracy, often proves to be difficult.

In Koliopanos et al. (2013) we used the Monte Carlo method to develop - for the first time - simulations of reflection from $\mathrm{C} / \mathrm{O}$ or $\mathrm{O} / \mathrm{Ne} / \mathrm{Mg}$, hydrogen poor disks. Perhaps paradoxically, our study demonstrated that the most striking effect of the hydrogen poor, $\mathrm{C} / \mathrm{O}$ rich material is not an appearance of strong fluorescent lines of carbon and oxygen - as one might expect - but nearly complete disappearance of the iron $\mathrm{K} \alpha$ line. This is caused by the "screening" of iron by the much more abundant carbon and oxygen. In optically thick, neutral material with solar abundances, the most likely process for incident photons with $\mathrm{h} v \gtrsim 7.1 \mathrm{keV}$ (Fe K-shell photo-ionisation threshold) is absorption by iron due to the photo ionisation of its atoms. The process is followed by the emission of fluorescent $\mathrm{Fe} \mathrm{K} \alpha$ photons at $6.4 \mathrm{keV}$ (with a fluorescence yield of approximately one third Bambynek et al. 1972). Consequently, the majority of photons with energies above this threshold will be absorbed by iron and will, therefore, contribute to its fluorescent line.

In the case of a $\mathrm{C} / \mathrm{O}$ (or $\mathrm{O} / \mathrm{Ne}$ ) white dwarf though, the extraordinary overabundance of oxygen makes it the dominant absorbing agent even at energies well beyond its own K-edge (see Fig. 1 in Koliopanos et al. 2013). As a result only a very small fraction of incident photons will fuel the iron $\mathrm{K} \alpha$ line. Despite the fact that the $\mathrm{O} \mathrm{K} \alpha$ line produced in this environment is boosted significantly, it is still strongly diluted by the primary continuum and therefore is difficult to detect. A much more visible effect is the significant attenuation or complete disappearance of the iron line. Helium, on the other hand, is not capable of screening iron, due to its smaller charge and correspondingly smaller absorption cross-section at the iron K-edge. Furthermore, helium is fully ionized throughout most of the accretion disk, for the entire range of accretion rates, observed in XRBs. Therefore in the case of a He-rich donor X-ray reflection is practically indistinguishable from the case of $\mathrm{H}$-rich accretion disks of "standard" XRBs, and the iron line has its nominal strength .

More specifically, for a source of moderate luminosity $\left(\mathrm{L}_{\mathrm{X}} \lesssim\right.$ a few $10^{37} \mathrm{erg} \mathrm{s}^{-1}$ ) the predicted suppression of the $\mathrm{Fe} \mathrm{K} \alpha$ line - in the case of a $\mathrm{C} / \mathrm{O}$ or $\mathrm{O} / \mathrm{Ne} / \mathrm{Mg}$ WD donor - translates to a more 
than an order of magnitude decrease of the EW of the line. On the other hand, in the case of a He-rich donor the iron line remains unaffected, i.e., with the EW near the typical value of the order of $\sim 100 \mathrm{eV}$ (e.g., Cackett et al. 2010; $\mathrm{Ng}$ et al. 2010). These predictions were observationally tested in Koliopanos et al. (2014) where we demonstrated that this effect can be used as a diagnostic of the chemical composition of the accretion disk and donor star of UCXBs.

In addition to the direct approach of spectral analysis, the composition of the accreted matter can be indirectly inferred by monitoring the systems' bursting activity in NS powered UCXBs (all known UCXBs host NSs). Type I X-ray bursts are the result of accumulation of $\mathrm{H}$ and/or He on the NS surface until it reaches the necessary conditions to ignite as a thermonuclear flash (e.g. Grindlay et al. 1976; Hansen \& van Horn 1975 and for a detailed review Strohmayer \& Bildsten 2006). Based on their recurrence rate and temporal characteristic (duration, light-curve) one can indicate the donor type chemical composition in UCXBs, from their bursting activity. However, it must be noted that the persistence and the rate of the accretion process are also crucial factors determining the bursting activity of X-ray binaries.

In this paper we build on the predictions of Koliopanos et al. (2013) and the findings of Koliopanos et al. (2014) in order to provide an assessment of the donor star type of all known UCXBs. We study X-ray spectra from various X-ray observatories for all known UCXBs and use our simulations to estimate the chemical composition of disk and donors star, based on the presence and strength of the iron $\mathrm{K} \alpha$ line. We evaluate our results by comparing with previous considerations, based on available optical analysis. We further account for the bursting activity of each source - based on the latest available monitoring information - contrasting their activity to our own estimations in order to scrutinize our classification. Our aim is to use X-ray spectroscopy to diagnose the chemical composition of the donor star of UCXBs, and provide a substantial addition to the study of the evolution of double degenerate systems.

\section{DATA SELECTION, EXTRACTION \& ANALYSIS}

We analyse all 14 confirmed UCXBs (i.e. with a measured optical period of less than $1 \mathrm{hr}$ ) plus 2 well known candidate UCXBs, albeit with tentative orbital period estimation (4U 0614+091, 4U 172834 ). We primarily use $X M M-N e w t o n$ observations since they provide the optimum combination of spectral resolution and effective area, in the $6-7 \mathrm{keV}$, to allow for clear line detection and EW estimation but more importantly to attain a tight upper limit in sources were no line is detected. For this reason we selected observations at high count rate. In case were XMM-Newton data were unavailable we made use of NUSTAR or RXTE observations. For all UCXBs we report their bursting activity (or lack there of), based on the archive all high energy all-sky monitors, such as the All Sky Monitor (ASM, on board RXTE), BeppoSAX, JEM-X (on board INTEGRAL), Fermi-LAT and MAXI. We reference the works of Jenke et al. (2016) and (Cumming 2003, for 4U 1820-30), Degenaar et al. (2013); Keek et al. (2017) and Strohmayer et al. (2018) for IGR J17062-6143, the Multi-INstrument Burst ARchive (MIN$\mathrm{BAR}^{1}$ ) and the SRON catalog of X-ray bursters ${ }^{2}$ (in 't Zand et al.). In Table 1 we present the list of sources analysed in this work, the

\footnotetext{
1 http://burst. sci . monash. edu/wiki/index . php?n=MINBAR . V09ReleaseNotes

2 https://personal.sron.nl/ jeanz/bursterlist.html
}

ObsIDs of the selected observations along with their distances and count rates.

\subsection{XMM-Newton spectral extraction}

For the XMM-Newton data, we only considered the EPIC-pn detector. EPIC-pn has the largest effective area of the three CCD detectors on board the XMM-Newton ( approximately five times higher than that of MOS detectors at $\approx 7 \mathrm{keV}$ ). Furthermore, all sources have very high count-rates registering $\approx 5 \times 10^{5}$ photons for each of the observations considered, thus providing sufficient statistics to robustly detect the presence of emission lines - or place tight EW upper limits when not detected. Using one type of detector ensures simplicity and self-consistency in our analysis. Therefore the following description of data analysis refers only to the EPIC-pn instrument.

The data were handled using the $X M M$-Newton data analysis software SAS version 18.0.0. and the calibration files released ${ }^{3}$ on March 29, 2019. Following the standard XMM-Newton guidelines, we filtered all observations for high background-flaring activity, by extracting high-energy light curves $(10<\mathrm{E}<12 \mathrm{keV})$ with a $100 \mathrm{~s}$ bin size. By placing appropriate threshold count rates for the highenergy photons, we filtered out time intervals that were affected by high particle background. All XMM-Newton observations considered for this work have been checked and for pile-up and were found to be unaffected. Only in the case of $4 \mathrm{U} 1820-30$, all available $X M M$-Newton observations suffered from pile-up, for this reason a recent NUSTAR observation was used for our analysis.

With the exception of 4U 0513-40 (ObsID:0151750101) and 4U 1850-087 (ObsID:0142330201), which were taken in full window Imaging mode, all other XMM-Newton observations had pn operating in Timing Mode. For the two sources observed in Imaging mode, spectra were extracted from a circular region with a radius $45^{\prime \prime}$ centered at the core of the point spread function (psf) of each source. We thus ensured the maximum encircled energy fraction $^{4}$ within the extraction region. The extraction and filtering process followed the nominal guidelines provided by the $X M M$ Newton Science Operations Centre ( $\left.\mathrm{SOC}^{5}\right)$. Spectral extraction was done with SAS task evselect using the standard filtering flags (\#XMMEA_EP \&\& PATTERN $<=4$ for pn), and SAS tasks rmfgen and arfgen were used to create the redistribution matrix and ancillary file, respectively. The spectra were regrouped to have at least 25 counts per bin and analysis in order to allow for the use if $\chi^{2}$ statistics in the spectral fitting. Source photons for all pn observations, taken in timing mode, were extracted from the RAW coordinate event file. More specifically the source spectra where extracted from 12 columns comprising the core source emission located between RAWX 25 and 50 for all sources. Background was extracted from RAWX 3 to 5, where RAWX is the coordinate along the column axis.

\subsection{NUSTAR spectral extraction}

For the analysis of the NUSTAR data, we used version 1.8.0 of the NuSTAR data analysis system (NuSTAR DAS) and instru-

\footnotetext{
3 XMM-Newton CCF Release Note: XMM-CCF-REL-367

4 See XMM-Newton Users Handbook \$3.2.1.1

http://xmm-tools.cosmos.esa.int/external/xmm_user_support/

documentation/uhb/onaxisxraypsf.html

5 http://www.cosmos.esa.int/web/xmm-newton/sas-threads
} 
Table 1. Observations analyzed in this work. UCXBs are tabulated along with the count-rates of selected observations, their most recent distance and orbital period estimations and their relevant references.

\begin{tabular}{lccccc} 
Source & $\begin{array}{c}\text { Distance } \\
(\mathrm{kpc})\end{array}$ & $\begin{array}{c}\text { Period } \\
(\text { minutes })\end{array}$ & Telescope & ObsID & $\begin{array}{c}\text { Net count rate } \\
\text { (cts/s) }\end{array}$ \\
\hline 4U 0513-40 & $12.1 \pm 0.6^{a}$ & $17^{b}$ & XMM-Newton & 0151750101 & $35.89 \pm 0.05$ \\
4U 0614+091 & $3.2 \pm 0.5^{c}$ & $51 ?^{d}$ & XMM-Newton & 0111040101 & $230.9 \pm 0.14$ \\
2S 0918-549 & $5.4 \pm 0.8^{e}$ & $17.4^{f}$ & XMM-Newton & 0061140101 & $55.45 \pm 0.04$ \\
XTE J0929-314 & $8_{-3}^{+7} g^{i}$ & $43.6^{h}$ & RXTE & 70096030500 & $30.12 \pm 0.14$ \\
4U 1543-624 & $7.0 ?^{i}$ & $18.2^{i}$ & XMM-Newton & 0061140201 & $208.7 \pm 0.07$ \\
& & & RXTE & P20071 & $382.42 \pm 0.98$ \\
4U 1626-67 & $8_{-3}^{+5}{ }^{j}$ & $42^{j}$ & XMM-Newton & 0111070201 & $28.03 \pm 0.05$ \\
& & & NuSTAR & 30101029002 & $12.42 \pm 0.08$ \\
IGR J17062-6143 & $7.3 \pm 0.5^{v}$ & $38^{v}$ & XMM-Newton & 0790780101 & $16.5 \pm 0.02$ \\
4U 1728-34 & $5.2 \pm 0.8^{k}$ & $10.8 ?^{k}$ & XMM-Newton & 0671180501 & $123.3 \pm 0.05$ \\
XTE J1751-305 & $8_{-1.3}^{+0.5}$ & $42^{m}$ & XMM-Newton & 0154750301 & $80.95 \pm 0.05$ \\
Swift J1756.9-2508 & $8.5 \pm 4^{n}$ & $54.7^{n}$ & XMM-Newton & 0830190401 & $19.45 \pm 0.09$ \\
& & & RXTE & P92050 & $21.51 \pm 0.08$ \\
XTE J1807-294 & $8_{-3.3}^{+4} g^{g}$ & $40.1^{o}$ & XMM-Newton & 0157960101 & $32.50 \pm 0.07$ \\
4U 1820-30 & $7.9 \pm 0.4^{a}$ & $11^{p}$ & NuSTAR & 90401323002 & $92.11 \pm 0.22$ \\
4U 1850-087 & $6.9 \pm 0.3^{a}$ & $20.6^{q}$ & XMM-Newton & 0142330501 & $43.52 \pm 0.07$ \\
4U 1916-05 & $9.3 \pm 1.4^{r}$ & $50^{s}$ & XMM-Newton & 0085290301 & $52.87 \pm 0.07$ \\
M15 X-2 & $10.4 \pm 0.5^{a}$ & $22.6^{t}$ & XMM-Newton & 0087350801 & $79.73 \pm 0.10$ \\
NGC 6440 X-2 & $8.5 \pm 0.4^{a}$ & $57.3^{u}$ & RXTE & 94044040600 & $19.46 \pm 0.012$ \\
\hline
\end{tabular}

References: ${ }^{a}$ Harris (2010); ${ }^{b}$ Zurek et al. (2009); ${ }^{c}$ Brandt et al. (1992); ${ }^{d}$ Shahbaz et al. (2008); ${ }^{e}$ in't Zand et al. (2005); ${ }^{f}$ Zhong \& Wang (2011); ${ }^{g}$ Galloway (2006); ${ }^{h}$ Galloway et al. (2002); ${ }^{i}$ Wang \& Chakrabarty (2004); ${ }^{j}$ Chakrabarty (1998); ${ }^{k}$ Galloway et al. (2010); ${ }^{l}$ Papitto et al. (2008); ${ }^{m}$ Markwardt et al. (2002);

${ }^{n}$ Krimm et al. (2007); ${ }^{o}$ Markwardt et al. (2003); ${ }^{p}$ Stella et al. (1987); ${ }^{q}$ Homer et al. (1996); ${ }^{r}$ Galloway et al. (2008); ${ }^{s}$ Walter et al. (1982); ${ }^{t}$ Dieball et al. (2005); ${ }^{u}$ Ortolani et al. (1994); ${ }^{v}$ Keek et al. (2017); Strohmayer et al. (2018).

mental calibration files from CalDB v20180312. We used the NUPIPELINE script to calibrate and clean the data, considering the standard settings reference in the NUSTAR observer's manual. We reduced internal high-energy background, and screened out all passages through the South Atlantic Anomaly (settings SAACALC $=3$, TENTACLE $=\mathrm{NO}$ and SAAMODE$=$ OPTIMIZED). Source and background spectra were extracted using the NUPRODUCTS routine and instrumental responses were then produced for each of the two focal plane CCD detectors (FPMA/B). The source spectrum was extracted from a circular region of 50" radius, and the background was estimated from a region of the same size, in a blank sky region on the same detector as the source, as far from the source as possible in order to avoid contribution from the PSF wings. Standard PSF, alignment and vignetting corrections were applied upon extraction.

\subsection{RXTE spectral extraction}

We used data from the RXTE mission for sources XTE J0929-314, Swift J1756.9-2508 and NGC 6440 X-2, for which no high quality data were available from other observatories. More specifically we obtained and analyzed spectra from the Proportional Counter Array (PCA, Jahoda et al. 2006) instrument on board RXTE. While PCA lacks the energy resolution of the CCD detectors on board XMMNewton and NUSTAR it has a very high effective area, yields spectra with very high $\mathrm{S} / \mathrm{N}$ ratio and is ideal for emission line-detection (and EW estimation), even if it cannot provide extremely accurate centroid energy or line-width estimation.

The PCA detector consists of five Proportional Counter Units (PCUs), with a combined effective area of $\sim 7000 \mathrm{~cm}^{2}$. Each PCU is composed of three layers of xenon (90\%) and methane (10\%) composites. The Incident photons with energies exceeding $20 \mathrm{keV}$ are mostly detected in the top layer (layer 1). We, therefore consider only spectra from this layer. Following the guidelines ${ }^{6}$ from the RXTE guest observer facility (GOF), we ignore energy channels below $3 \mathrm{keV}$ recommendation. We also removed all channels above $30 \mathrm{keV}$. Signal-to-noise ratio drops significantly above $\sim 30 \mathrm{keV}$, and for this work we are only interested in the energy range below $30 \mathrm{keV}$.

We filtered out observation intervals where the elevation angle was less than 10 degrees (to avoid possible Earth occultations) and also any data that may have been received during passage from the South Atlantic Anomaly. Furthermore, we excluded any data affected by electron contamination, were offset by more than 0.02 degrees or taken 150 seconds before, through 600 seconds after any PCU2 breakdowns, during the observation. Source spectra were extracted and background emission was modeled using standard routines from the FTOOLS package and the latest model for bright sources, provided by the $\mathrm{GOF}^{7}$.

\subsection{Spectral analysis}

The X-ray spectral analysis was performed using the Xspec spectral fitting package, version 12.9.0 (Arnaud 1996). For the continuum fitting we used simple models consisting of the fewest possible free parameters. These were the xspec model diskbb for a multicolor disk black body (MCD), the spherical black body model (bbodyrad) for thermal emission components and a simple powerlaw model (with occasional exponential cutoff) for non-thermal emission. Despite their simplicity, all models are physically motivated. The MCD component is expected to originate in the accretion disk and the power law simulates non-thermal emission from a hot electron corona (e.g., Done et al. 2007; Gilfanov 2010). The additional bbodyrad component was employed to model thermal

\footnotetext{
6 https://heasarc.gsfc.nasa.gov/docs/xte/pca/doc/rmf/pcarmf-11.7/

$7 \mathrm{http}$ //heasarc.gsfc.nasa.gov/docs/xte/pca_news.html
} 
Table 2. Best fit parameters for the continuum emission spectra. 4U 1626-67 is the only UCXB that is a high-B NS, X-ray pulsar and is tabulated separately. All errors are in the $1 \sigma$ confidence range.

\begin{tabular}{|c|c|c|c|c|c|c|c|c|c|}
\hline Source & $\begin{array}{c}\mathrm{nH} \\
\times 10^{22}\end{array}$ & $\begin{array}{c}\mathrm{kT}_{\mathrm{dBB}} \\
\mathrm{keV}\end{array}$ & $\begin{array}{c}\mathrm{R}_{\mathrm{dBB}^{a}} \\
\mathrm{~km}\end{array}$ & $\Gamma$ & $\begin{array}{c}\mathrm{K}_{\mathrm{po}}{ }^{b} \\
{\left[\times 10^{-3}\right]}\end{array}$ & $\stackrel{\mathrm{kT}_{\mathrm{BB}}}{\quad \mathrm{keV}}$ & $\begin{array}{l}\mathrm{R}_{\mathrm{BB}}^{c} \\
\mathrm{~km}\end{array}$ & $\begin{array}{c}\mathrm{L}^{\mathrm{d}} \\
\times 10^{36} \mathrm{erg} \mathrm{s}^{-1}\end{array}$ & red. $\chi^{2} / d o f$ \\
\hline $4 \mathrm{U} 0513-40^{e}$ & $0.22 \pm 0.01$ & $0.11 \pm 0.001$ & $632 \pm 31.4$ & $2.01 \pm 0.01$ & $23.3 \pm 0.65$ & - & - & 2.24 & $1.12 / 163$ \\
\hline $4 \mathrm{U} 0614+091^{e}$ & $0.50 \pm 0.01$ & $0.10 \pm 0.001$ & $3526 \pm 702$ & $2.21 \pm 0.01$ & $306 \pm 1.11$ & - & - & 5.32 & $1.16 / 1888$ \\
\hline 2S 0918-549 & $0.07 \pm 0.001$ & $0.59 \pm 0.01$ & $15.3 \pm 2.28$ & $0.96_{-032}^{+0.22}$ & $3.23_{-174}^{2.23}$ & $1.23 \pm 0.03$ & $0.57 \pm 0.09$ & 0.68 & $1.08 / 1848$ \\
\hline XTE J0929-314 & $0.12^{f}$ & $0.57 \pm 0.12$ & $85.1_{-54.6}^{+126}$ & $1.88 \pm 0.08$ & $89.5_{-16.1}^{-1.14}$ & - & - & 3.69 & $0.48 / 49$ \\
\hline $4 \mathrm{U} 1543-624^{e}$ & $0.29^{f}$ & $0.38 \pm 0.12$ & $14.2_{-0.56}^{+0.64 g}$ & - & - & $1.53 \pm 0.01$ & $0.16 \pm 0.03$ & 3.64 & $1.03 / 1795$ \\
\hline IGR J17062-6143 & $0.14 \pm 0.01$ & $0.50 \pm 0.03$ & $14.5 \pm 1.04$ & $1.22_{-0.47}^{+0.35}$ & $1.28_{-0.85}^{1.60}$ & $1.00_{-0.10}^{+0.08}$ & $0.49 \pm 0.02$ & 0.36 & $1.14 / 175$ \\
\hline $4 \mathrm{U} 1728-34$ & $2.25 \pm 0.01$ & $0.72 \pm 0.03$ & $10.7 \pm 1.64$ & - & - & $2.27 \pm 0.01$ & $0.10 \pm 0.01$ & 5.70 & $1.18 / 1748$ \\
\hline XTE J1751-305 & $1.39 \pm 0.02$ & $0.90 \pm 0.01$ & $9.89_{-0.56}^{+1.46}$ & - & - & $3.45 \pm 0.01$ & $0.49 \pm 0.01$ & 8.21 & $1.05 / 1695$ \\
\hline Swift J1756.9-2508 & $5.47 \pm 0.03$ & $0.94 \pm 0.07$ & $10.6 \pm 1.11$ & - & - & $3.20_{-0.16}^{+0.20}$ & $0.41 \pm 0.05$ & 5.05 & $1.04 / 1859$ \\
\hline XTE J1807-294 ${ }^{e}$ & 0.57 & $0.59 \pm 0.01$ & $11.7_{-3.36}^{+5.83}$ & - & - & $2.73 \pm 0.10$ & $0.38 \pm 0.01$ & 1.84 & $1.01 / 1632$ \\
\hline $4 \mathrm{U} 1820-30$ & $0.14^{f}$ & $0.67_{-0.06}^{+0.05}$ & $192 \pm 9.74$ & $2.12_{-0.41}^{+0.57^{h}}$ & $665_{-32.3}^{31.8}$ & - & - & 65.4 & $1.00 / 589$ \\
\hline $4 \mathrm{U} 1850-087^{e}$ & $0.25^{f}$ & $0.45 \pm 0.01$ & $16.7 \pm 0.74$ & - & - & $1.96 \pm 0.04$ & $0.54 \pm 0.02$ & 14.4 & $1.19 / 161$ \\
\hline 4U 1916-05 & $0.53 \pm 0.02$ & $0.11 \pm 0.001$ & $859 \pm 41.3$ & $1.93 \pm 0.03$ & $64.5_{-1.43}^{1.46}$ & $1.59 \pm 0.05$ & $0.59 \pm 0.12$ & 8.96 & $1.09 / 1797$ \\
\hline 4U 2129+11 (M15 X-2) & $0.20 \pm 0.02$ & $0.10_{-0.02}^{+0.01}$ & $354 \pm 53.2$ & $1.45 \pm 0.02$ & $44.6_{-0.96}^{0.113}$ & - & - & 11.7 & $1.05 / 1711$ \\
\hline NGC 6440 X-2 & $0.31^{f}$ & - & - & $1.85 \pm 0.03$ & $62.6_{-4.52}^{4.840}$ & - & - & 7.34 & $0.48 / 35$ \\
\hline $4 \mathrm{U} 1626-67^{e}$ & $0.12 \pm 0.01$ & $0.36 \pm 0.01$ & $95.8_{-3.70}^{+9.15}$ & $0.72 \pm 0.01$ & $6.04_{-0.05}^{0.06}$ & - & - & 162 & $1.04 / 162$ \\
\hline
\end{tabular}

${ }^{a} R_{\mathrm{dBB}}$ (the inner radius of the accretion disk in km) is inferred from diskbb model, by solving $\mathrm{K}_{\mathrm{dBB}}=\left(\mathrm{R}_{\mathrm{dBB}} / \mathrm{D}_{10}\right)^{2} \cos i$, for $R_{\mathrm{dBB}}$. ' $\mathrm{K}_{\mathrm{BB}}$ ' is the normalisation of the diskbb model, $\mathrm{D}_{10}$ is distance in units of $10 \mathrm{kpc}$ and $i$ is the inclination. In all sources this was assumed to be $60^{\circ}$, with the exceptions of $4 \mathrm{U} 1916-05$ and $4 \mathrm{U}$ 0513-40 (where $i$ is set to $85^{\circ}$ ), which are known edge-on viewed systems (Boirin et al. 2004; Fiocchi et al. 2011). With the exception of XTE J19029-314 (for which the K estimation has considerably high $1 \sigma$ error bars), errors in radius estimation are dominated by the $1 \sigma$ errors in the distance estimation (see Table 1).

${ }^{b}$ Power-law component normalisation constant: photons $/ \mathrm{keV} / \mathrm{cm}^{2} / \mathrm{s}$ at $1 \mathrm{keV}$

${ }^{c}$ Size of the spherical black body component (bbodyrad model), estimated from $\mathrm{K}_{\mathrm{BB}}=\mathrm{R}_{\mathrm{BB}}{ }^{2} / \mathrm{D}_{10}{ }^{2}$, where $\mathrm{K}_{\mathrm{BB}}$ is the normalization parameter of the bbodyrad model.

${ }^{d}$ Luminosity in the $0.5-10 \mathrm{keV}$ range, extrapolated from the best-fit model.

${ }^{e}$ Emission and/or absorption-like features detected $<1 \mathrm{keV}$ were modelled using a combination of Gaussian absorption lines. For justification and details see Section 3.4.

${ }^{f}$ Parameter frozen at total galactic H I column density (HI4PI Collaboration et al. 2016).

$g$ There is considerably uncertainty in the distance estimation for this source. Radius is estimated for a distance of $7 \mathrm{kpc}$ (Wang \& Chakrabarty 2004 ). Error bars are estimated from the $1 \sigma$ errors for the best-fit value of $K_{\mathrm{dBB}}$.

${ }^{h}$ With exponential cutoff at high energies see Section 3.4 for details.

emission from the NS surface, known as boundary layer emission (e.g. Popham \& Sunyaev 2001).

When detected, the $\mathrm{Fe} \mathrm{K} \alpha$ emission line is modeled using a simple Gaussian model, based on which we estimate the EW. For consistency check, we estimated the EW of the emission line in two ways: a) For the broadband continuum fit. b) For a fit of only the 4-8 $\mathrm{keV}$ spectral range - where the continuum is modelled using a smooth power-law. We found that both estimations were consistent, within $1 \sigma$ error bars and we, therefore, opted to report the EW value of the broadband fits. In case of non-detection we estimate the $1 \sigma$ upper limit on the EW of a Gaussian emission line with a width between $10-500 \mathrm{eV}$ and a centroid energy between 6.4-6.9 keV. More specifically, we produce a range of EW upper limits of a grid of emission lines with the centroid energy in the 6.4-6.9 $\mathrm{keV}$ with a step of $0.1 \mathrm{keV}$ and width within the $10-500 \mathrm{eV}$ range with a step of $20 \mathrm{eV}$. We report the average value of all EW upper limit estimations.

Due to the non-solar abundance of the accreted material in UCXBs, their spectra are often interspersed with narrow absorption and/or emission-like features in the soft $(<1 \mathrm{keV}) \mathrm{X}$-rays (e.g., Paerels et al. 2001; Juett et al. 2001). They are likely the result of emission by overabundant elements such as $\mathrm{C}$ and $\mathrm{O}$ but also pronounced absorption edges from highly non-solar circum-source material. A meticulous study of these features would involve modeling the interstellar absorption by adding a secondary absorption component for the circum-source material assuming non-solar abundances of elements, along with high resolution analysis of the emission spectra (using e.g. RGS). Such an endeavour is out- side of the scope of this work. For this reason all absorption or emission-like residuals below $\sim 2 \mathrm{keV}$ where modelled, empirically using Gaussian absorption and emission lines and in some cases (see below) non-solar interstellar absorbers. No attempt was made to exact any physical interpretation of such features and they were only modelled in order to ensure a robust description of the observed spectra. In most cases, dedicated analysis of these features has been done by previous authors. We refer to these works in all relevant segments of our text.

\subsection{Source specific comments}

Below we briefly describe the spectral analysis of the sources in our UCXB catalog. When thermal emission is detected we convert the model normalization parameter into inner disk radius (for the diskbb model) and size of emitting region (for the bbodyrad). These estimations depend on the source distance - and the error bar estimation is dominated by the distance estimation. All current distance estimations are presented in Table 1. The interstellar absorption was modeled using the improved version of the tbabs code ${ }^{8}$ (Wilms et al. 2000). The atomic cross-sections were adopted from Verner et al. (1996). We have divided the sources into two groups based on the presence or absence of the iron $\mathrm{K} \alpha$ emission line. To allow for a more concise reading experience, we do not describe the spectral fitting results of each individual source, but rather summarise the process for the whole sample. All best-fit values for the

\footnotetext{
${ }^{8}$ http://pulsar.sternwarte.uni-erlangen.de/wilms/research/tbabs/
} 
continuum fitting are presented in Table 2. All estimations for the iron $\mathrm{K} \alpha$ emission line are tabulated in Table 3.

In this table we use the notation "Soft" for sources where thermal emission is either the sole component or has a $0.5-10 \mathrm{keV}$ flux at least three times higher than the non-thermal component, and "Hard" for spectra dominated by non-thermal emission (using the same rule). The two notations allude to the so-called "Soft" and "Hard states" of XRBs (e.g. see review by Gilfanov 2010). We note that the state transition of XRBs are much more nuanced than a simple division into soft and hard states (e.g., in our two "transitioning" sources the two components have comparable flux) and that these characterisations are used to briefly denote the dominant spectral component in Table 3.

\subsubsection{Sources WITH a Fe Ko emission line}

In sources 4U 0614+091, IGR J17062-6143, 4U 1728-34, 4U 182030, 4U 1916-05 and NGC 6440 X-2 we detect a prominent Fe K $\alpha$ line with and EW ranging from $\sim 50$ to $\sim 240 \mathrm{eV}$. In all sources the emission-like residuals on top of the data-to-model ratio plot, were prominent enough to allow for easy visual confirmation (Figure 1, left panel). The addition of a Gaussian emission feature on all sources yielded a $\delta \chi^{2}$ value of over 50 for 3 dof, indicating more than $6 \sigma$ significance of detection. In several sources we also noted the presence of narrow residuals in the 1.7-2.5 kev range. They, most likely originate from incorrect modeling of the Si and $\mathrm{Au}$ absorption in the CCD detectors by the EPIC pn calibration (e.g. Ng et al. 2010), which often results in the presence of emission and/or absorption features at $\sim 1.84 \mathrm{keV}, \sim 2.28 \mathrm{keV}(\mathrm{M} \beta)$ and $2.4 \mathrm{keV}(\mathrm{M} \gamma)$. The features are not very pronounced and do not affect the quality of the fit or the aim of our analysis. Therefore, we have ignored them, but their respective energy channels are still included in our fits.

In sources 4U 0614+091 and 4U 1728-34 we also detected emission (at $\sim 0.5 \mathrm{keV}$ ) and absorption-like (at $\sim 0.7 \mathrm{keV}$ ) features which where modelled using Gaussian lines. In 4U 1916-05 we further detect narrow absorption-like features at $\sim 6.65$ and $\sim 6.95 \mathrm{keV}$ that are consistent with resonant absorption from FeXXV and FeXXVI ions, respectively, in agreement with the findings of Juett \& Chakrabarty (2006), using Chandra data. The best-fit values for the continuum are presented in Table 2 and for the iron $\mathrm{K} \alpha$ emission lines in Table 3, along with the bursting activity (or lack thereof) of each source.

\subsubsection{Sources WITHOUT a Fe Ka emission line}

We found no evidence of iron line emission for sources 4U 051340, 2S 0918-549, XTE J0929-314, XTE J1751-305, XTE J1807294, 4U 1850-087, M15 X-2 (4U 2129+11 ${ }^{9}$ ). For all sources we estimated the $1 \sigma$ upper limits on the EW emission line, which we tabulate in Table 3. We also noted a prominent emission-like feature at $\sim 1 \mathrm{keV}$ in source XTE J1807-294. Data-to-model ratio vs energy plots are presented in Fig. 1, right panel.

\subsubsection{Sources with a variable Fe Ka emission line}

In the 2001 XMM-Newton observation of 4 U 1543-624 no iron emission was detected in the $6-7 \mathrm{keV}$ region, with a tight $\mathrm{EW}$ upper limit of $\sim 3 \mathrm{eV}$. The source was in the soft state, characterized

9 Not to be confused with neighboring source 4 U $2129+12$ by thermal emission which we model using a combination of MCD and spherical black body models. Analysis of older (1997) RXTE observations, revealed the presence of a prominent Fe K $\alpha$ emission line (see also analysis by Schultz 2003) for a similar continuum. Swift J1756.9-2508 was also dominated by thermal emission with no iron emission line detected with an $1 \sigma$ upper limit of $\sim 5 \mathrm{eV}$ on the EW of a Gaussian emission line. However, in previous outbursts in 2007 and 2009 a very prominent iron emission line was detected in RXTE spectra of Swift J1756.9-2508 (see Patruno et al. 2010, for the iron emission in the 2009 observation). We have also analyzed the 1997 RXTE observation of 4U 1543-624 and the 2007 of Swift J1756.9-2508, which we present in Table A1 in the appendix. The variability of the line in the two sources is mentioned here and briefly discussed in Section 4, but is thoroughly examined in a separate publication (Koliopanos et al. accepted by MNRAS).

Apparent, complex residuals were detected in the sub- $1 \mathrm{keV}$ spectral region of 4U 1543-624 and were modelled using two emission and one absorption Gaussian component. Due to degeneracy between the interstellar absorption column density value and the parameters of the absorption lines, the column density was frozen to its galactic value (indeed, proper modelling of the line-of-sight absorption in $4 \mathrm{U}$ 1543-624 requires additional local absorption with extra-solar abundances, see e.g. Juett et al. 2001). Best-fit values of the spectral continuum of the sources are found in Table 2 and EW upper limits of the $\mathrm{Fe} \mathrm{K} \alpha$ line, along with bursting activity in Table 3. The variable presence of the iron line of the two sources is depicted in their data-to-model ratio vs energy plots in Fig. 2.

The third source with apparent iron line variability is 4U 162667 which is unique among UCXBs, as it is the only source powered by a highly magnetized $\left(\mathrm{B} \sim 10^{12} \mathrm{G}\right) \mathrm{NS}$ and is an X-ray pulsar. In this analysis we used a $2003 X M M$-Newton observation. The source continuum was fitted with a power-law with a spectral index of $\sim 0.8$ and an exponential cutoff at $\sim 8 \mathrm{keV}$ and an MCD component with $\mathrm{kT} \sim 0.4 \mathrm{keV}$. Narrow emission-like feature were also detected at $\sim 0.6 \mathrm{keV}$ and $\sim 1 \mathrm{keV}$ and were modelled using Gaussian emission lines. No iron line emission was detected during this observation. Nevertheless, after its 2010 luminosity increase and torque reversal, a faint emission $\mathrm{Fe} \mathrm{K} \alpha$ emission line was detected in RXTE and Chandra data (e.g. Koliopanos \& Gilfanov 2016). The Koliopanos \& Gilfanov findings were confirmed by a 2015 NuSTAR observation (e.g. Iwakiri et al. 2019). We also analyze this observation and tabulate our findings in Table A1 in the Appendix. In the NUSTAR observation (which was also taken during the more luminous spin-up era) we detect a moderately bright iron emission line with an EW value of $\sim 30 \mathrm{eV}$.

We note that due to the nature of this source - and despite our selection of the MCD model for the thermal emission in both observations - it is likely that it does not originate in the accretion disk and the iron line emission line is not the result of the standard coronal-disk reflection scenario modeled in Koliopanos et al. (2013). For these reasons, this source is discussed separately in Section 4 and its data-to-model ratio plot appears separately in Figure 3, for both epochs. One of the most well monitored X-ray binaries, $4 \mathrm{U}$ 1626-67 has never been observed to produce X-ray bursts.

\section{DISCUSSION}

\subsection{The spectral continuum}

We have revisited X-ray observations of all known UCXBs. Our goal was not to retrace previous spectral analyses, but rather to 

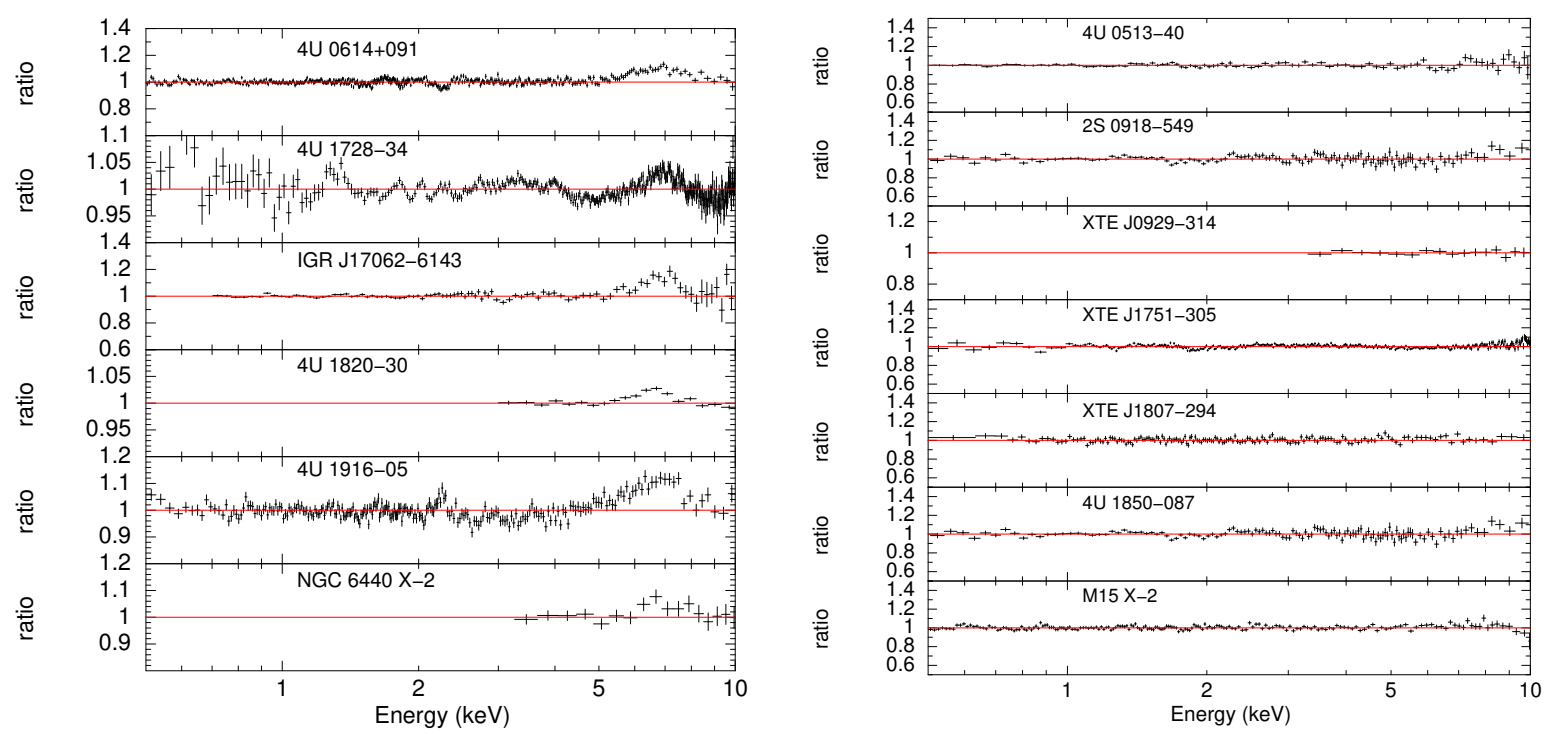

Figure 1. Left: Data-to-model ratio plots for all sources with a detected Fe K $\alpha$ emission line. The model includes all fit-required components (i.e. thermal and non-thermal continuum emission, soft X-ray emission features), except the Gaussian component used to model the iron emission. Right: Data-to-model ratio plots for all sources with no iron line detection. All sources are included except for 4U 1626-67. Due to its unique nature - as an UCXB, X-ray pulsar - the analysis of this source is presented separately.

Table 3. Final results for the ultracompact X-ray binaries sample.

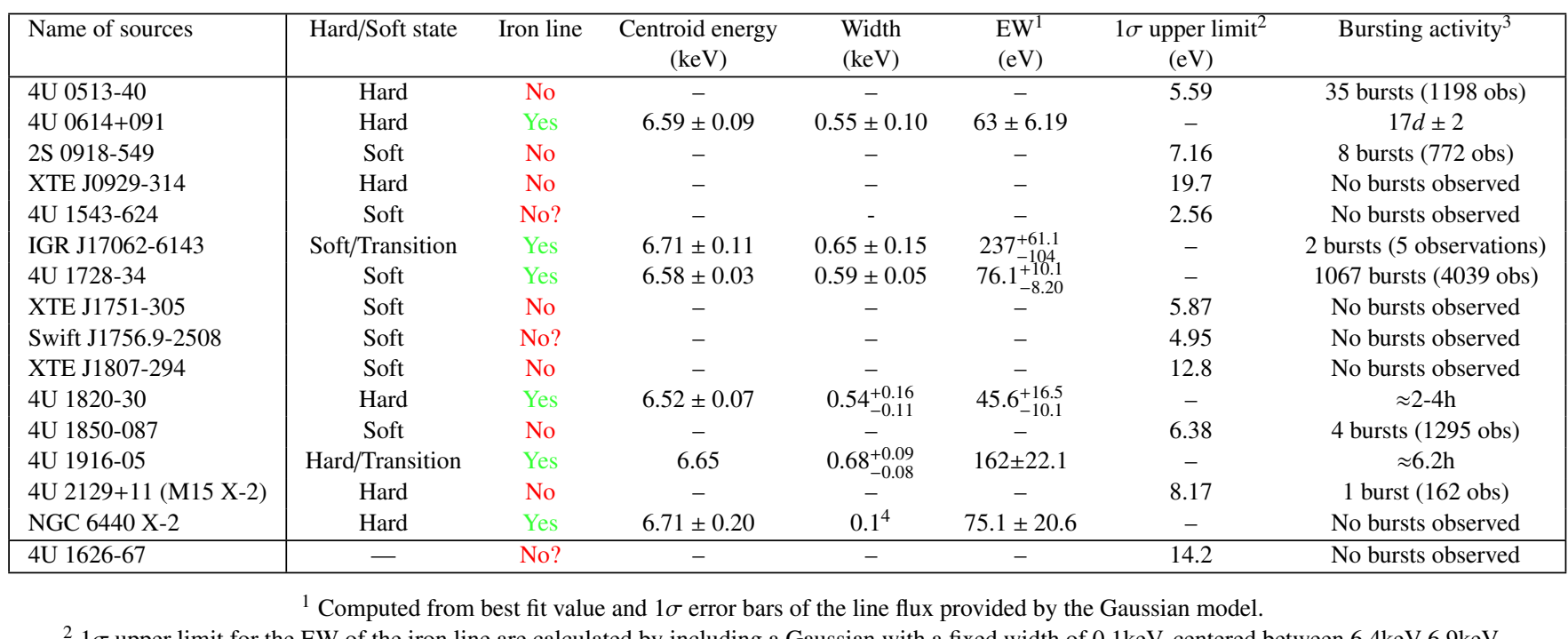

${ }^{2} 1 \sigma$ upper limit for the $\mathrm{EW}$ of the iron line are calculated by including a Gaussian with a fixed width of $0.1 \mathrm{keV}$, centered between $6.4 \mathrm{keV}-6.9 \mathrm{keV}$.

${ }^{3}$ From Jenke et al. (2016) and Cumming (2003) (4U 1820-30) for recurrence times. From Degenaar et al. (2013) and Keek et al. (2017); Strohmayer et al. (2018) for IGR J17062-6143 and van Paradijs et al. (1990) for 4U 2129+11. From http://burst.sci.monash.edu/wiki/index.php?n=MINBAR.VQ9ReleaseNotes for number of bursts, and from https://personal.sron.nl/ jeanz/bursterlist.html for the rest.

4 This parameter was frozen.

model the spectral continuum in a consistent and physically motivated manner, in order to draw conclusions on the presence or absence of the primary X-ray reflection feature in X-ray spectra: the iron $\mathrm{K} \alpha$ line. We then use our findings as a diagnostic of the chemical composition of their disk and donor star. Our analysis of the spectral continuum confirms a physically credible interpretation of the spectral shape for NS-XRBs, that has been noted in numerous previous studies (e.g. Barret 2001; Lin et al. 2007). From our sample of the sixteen known UCXBs we found eight sources in the "Soft" state, i.e. dominated by hot two thermal component (for sources 2S 0918-549 and IGR J17062-6143 a faint power law tail is also detected), while the other eight were found in the "Hard" state, dominated by non-thermal emission and a cool MCD component (4U 1916-05 also features a hot thermal component, signifying a state transition).

During the soft state of accreting highly magnetized neutron stars, the accretion disk is expected to reach the NS surface. Indeed, all sources in these state yield best fit values for the inner disk radius in the $10-15 \mathrm{~km}$ range. A hot accretion disk extending to the surface of the NS. Furthermore, in all spectra we detect a secondary hotter 


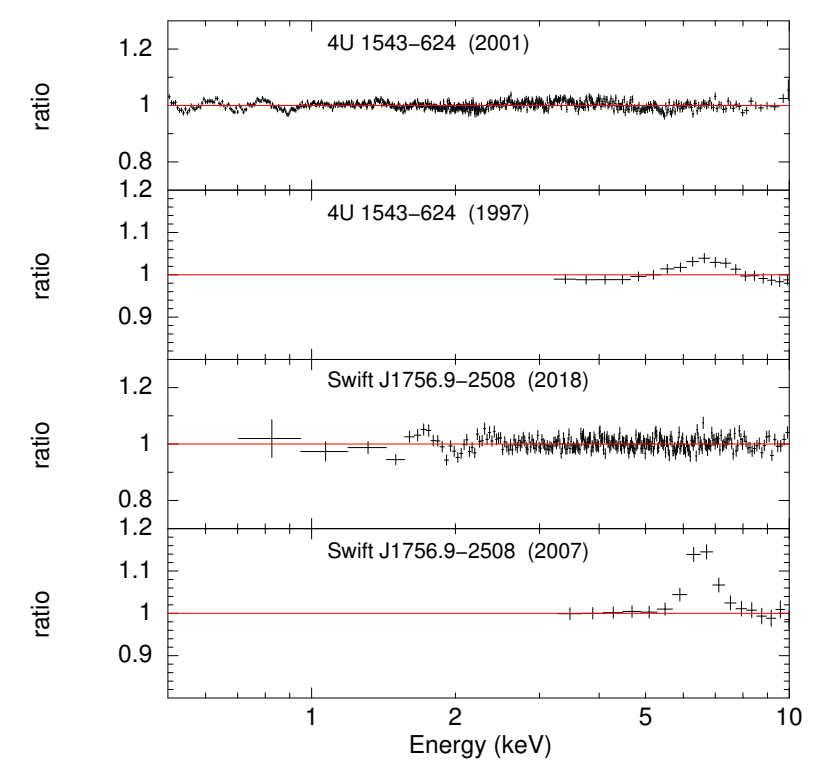

Figure 2. Data-to-model ratio plots for the two sources with a variable $\mathrm{Fe}$ $\mathrm{K} \alpha$ emission line.

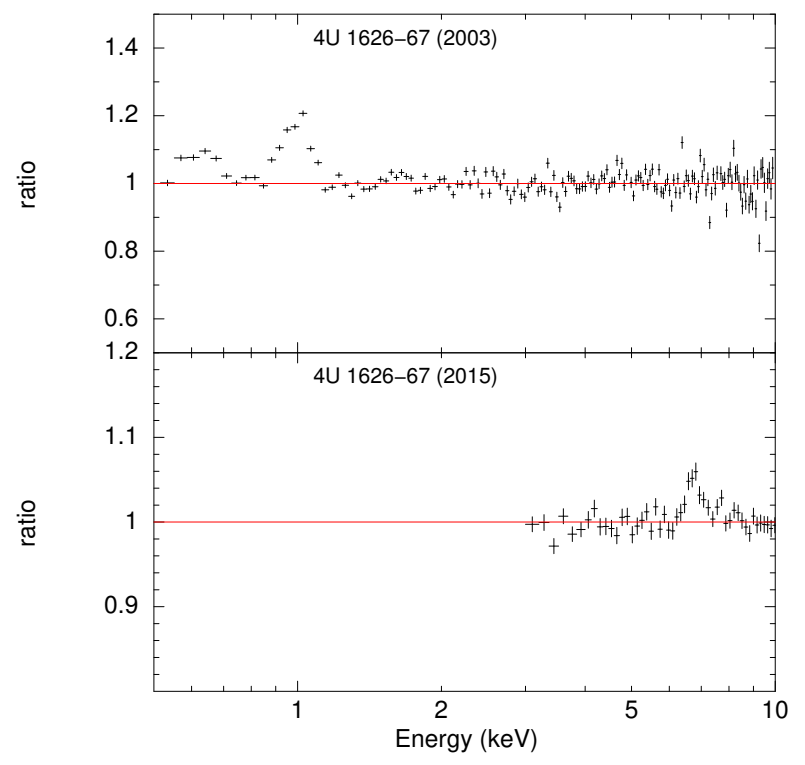

Figure 3. Data-to-model ratio plots for the variable $\mathrm{Fe} \mathrm{K} \alpha$ emission line detection in X-ray pulsar 4U 1626-67.

thermal component $(\mathrm{kT} \gtrsim 1 \mathrm{keV})$ consistent with emission from the hot, optically thick boundary layer expected to form on the surface of the NS during this state. Non-thermal tails during this state are usually detected in spectra from telescopes that extend at energies above $10 \mathrm{keV}$. The spectra of all sources found in the hard state were dominated by non-thermal emission, but also featured a less pronounced, but still significant soft MCD component. The accretion disk temperature of hard-state sources is considerably lower than that of soft-state sources, and the inner radius ten to hundred of times larger. These estimations are consistent with theoretical predictions of a truncated disk in hard state XRBs. The realistic quantities we derive from our best-fit values, increase the confidence in out analysis of the spectral continuum and consequently our estimations for the $\mathrm{EW}$ of the $\mathrm{Fe} \mathrm{K} \alpha$ line.

\subsection{Iron emission and X-ray diagnostics}

We have detected prominent and broad iron $\mathrm{K} \alpha$ emission lines in six of the known UCXBs (see Figure 1, left). For all sources for which the emission line was detected - and for which additional data with adequate statistics were available (see appendix for extra observations that were checked) - we determined the stable presence of the emission line in their spectra. Another seven sources in the UCXB catalog showed no evidence of iron line emission (Figure 1, right) with tight upper limits on iron emission, in most case $\mathrm{EW}<10 \mathrm{eV}$. Similarly to the previous sample, all other available observations were inspected in order to confirm the absence of the Fe $\mathrm{K} \alpha$ line. The remaining three known UCXBs exhibited a variability in the presence of the iron line (Figures 2 and 3 ).

\subsubsection{Non detection}

With the help of our Monte Carlo simulation from Koliopanos et al. (2013), we can use the results of our analysis on the iron line detection, in order to evaluate the chemical composition of the accretion disk and donor star. The code models fluorescence $\mathrm{K} \alpha$ and $\mathrm{K} \beta$ lines for elements between $\mathrm{Z}=3$ and 30 and estimates their EWs with respect to the total emission, which is a combination of both the primary and the reflected component. For this work we have updated our code with the abundances by (Wilms et al. 2000) ${ }^{10}$ and atomic cross-sections from Verner et al. (1996), for consistency with the xspec modelling. The code simulates the abundances of $\mathrm{C} / \mathrm{O}$-rich disk by "converting" $\mathrm{H}$ and $\mathrm{He}$ into $\mathrm{C}$ and $\mathrm{O}$. In practice we gradually reduce the solar-like abundances (by mass) of $\mathrm{H}$ and $\mathrm{He}$, while increasing those of $\mathrm{C}$ and $\mathrm{O}$ by the same amount. The total number of nucleons is conserved, mass ratios of all other elements are kept fixed at their solar values and the $\mathrm{C} / \mathrm{O}$ abundance ratio, also remains fixed. The position along this sequence of abundances can be give by the $\mathrm{O} / \mathrm{Fe}$ ratio normalized to its solar value from (Wilms et al. 2000). The value of the $\mathrm{O} / \mathrm{Fe}$ ratio corresponding to full "conversion" of $\mathrm{H}$ and $\mathrm{He}$ to $\mathrm{C}$ and $\mathrm{O}$ is 78 .

To evaluate the absence of the iron emission line in the spectra of our sources, we estimated the minimum value of the $\mathrm{O} / \mathrm{Fe}$ ratio, in order to produce an $\mathrm{Fe} \mathrm{K} \alpha$ line with an $\mathrm{EW}$ of $20 \mathrm{eV}$, which is the highest upper limit in our source sample with no iron detection (source: XTE J0929-314), for an incident power law with $\Gamma=1.9$ (best-fit parameter for XTE J0929-314). Assuming an angle of $80^{\circ}$ for the incident spectrum - which yields the lowest iron line EW for a given $\mathrm{O} / \mathrm{Fe}$ ratio - we deduce that an $\mathrm{O} / \mathrm{Fe}$ ratio of at least 12 is necessary in order to yield an iron emission line with $\mathrm{EW}<20 \mathrm{eV}$. The $\mathrm{O} / \mathrm{Fe}$ ratio increases to 25 for a face-on incident spectrum $\left(0^{\circ}\right)$. Incident spectra with lower spectral index or thermal primary emission (sources with thermal boundary layer emission at $\mathrm{kT} \gtrsim 1 \mathrm{keV}$ ), require $\mathrm{O} / \mathrm{Fe}>34$ in order for the iron line to be detected in their spectra at an $\mathrm{EW}<20 \mathrm{eV}$. Based on these estimations, the disappearance of the iron emission line from the spectra of the seven UCXBs: 4U 0513-40, 2S 0918-549, XTE J0929-314, XTE J1751305 , XTE J1807-294, 4U 1850-087 and 4U 2129+11 is the result of an $\mathrm{O} / \mathrm{Fe}$ ratio at least an order of magnitude higher than the solar value, which can be interpreted as the result of a $\mathrm{C} / \mathrm{O}$ or $\mathrm{O} / \mathrm{Ne} / \mathrm{Mg}$ donor in these sources.

10 With abundances from Anders \& Grevesse (1989) for elements not included in Wilms et al. 


\subsubsection{Persistent Ko emission line}

Six sources in the UCXB catalog feature a persistent iron emission line in their spectra, detected in multiple past observations (see Table 3 for the sources with $\mathrm{Fe} \mathrm{K} \alpha$ line detection and Table A2 in Appendix for alternative observations that we have inspected. Based on the findings of our simulations, if the iron emission lines are the result of disk reflection - further supported by the fact that all lines are considerably broadened - the disk and donor star must be dominated by helium. We also note two of the three sources that are also found in the soft-state feature an emission line with a considerably higher value of the EW (sources IGR J17062-6143 and 4 U 1916-05). Our estimations (see e.g. Table 2 in Koliopanos et al. 2013 ) indicate that soft-state sources produce emission lines with higher $\mathrm{EW}$ values (i.e. in the $150-250 \mathrm{eV}$ range). This is primarily due to the shape of the incident spectrum - i.e. a power law with $\Gamma \gtrsim 1.51$ vs a black body with $\mathrm{kT} \sim 1.5-3 \mathrm{keV}$ and is the result of higher available photons near the $7.1 \mathrm{keV} \mathrm{K}$-shell absorption edge of iron. However, harder power-law shaped incident spectra (e.g. $\Gamma<1$, usually found in accretion column emission) can produce Fe K $\alpha$ line with similar EW to the black body incident spectrum. While, the seeming agreement between our observational analysis and the simulation results is encouraging, it must be considered very cautiously, as there are several other parameters involved, including the disk-corona geometry, viewing angle and disk ionisation state.

\subsubsection{Variable presence of the Fe Ko emission line}

Examination of the rich observational archive of the UCXB source catalog revealed three sources for which the presence of the iron $\mathrm{K} \alpha$ line varies through the years. This is a very intriguing discovery, since the variability of this process - i.e. screening of the iron line by carbon and oxygen - had been predicted by our initial analysis in Koliopanos et al. (2013). More specifically, in C/O dominated disks the screening efficacy of the two elements depends entirely on whether they are fully ionized or not. When oxygen (and therefore carbon) become fully ionized in the disk, the EW of the iron line is expected to return to its initial value, which is largely determined by the abundance of iron in the disk. In the Koliopanos et al. paper, simple analytical arguments were used to predict a luminosity dependence of the iron line variability. Under the assumption that the ionization state of the disk mostly depends on the accretion rate, it was predicted that a critical luminosity value for the variability of the iron line presence was at $\sim 10^{37} \mathrm{erg} / \mathrm{s}$, in the $0.5-10 \mathrm{keV}$ range. Nevertheless, sources 4U 1543-624 and Swift J1756.9-2508 were observed at similar luminosity $\left(<10^{37} \mathrm{erg} / \mathrm{s}\right)$ and at similar spectral states withe the $\mathrm{Fe} \mathrm{K} \alpha$ line being the only emission component that varied dramatically between observations. Therefore, a detailed study of these two sources and the conditions of the iron line variability is needed in order to interpret the phenomenon. Such an analysis was indeed conducted and is presented in a separate work by Koliopanos et al. (accepted by MNRAS).

The third source with a variable presence of the iron line is $4 \mathrm{U}$ 1626-67. This is an exceptional source - even within the already extraordinary UCXB population, as it is the only UCXB which has a highly magnetized NS accretor. It is a persistent X-ray pulsar with a known magnetic field of $\sim$ a few $\times 10^{12} \mathrm{G}$, based on detection of cyclotron resonant scattering features, (e.g. Iwakiri et al. 2019). The iron line variability on this source was first reported by Koliopanos \& Gilfanov (2016). Using archival data it was demonstrated that the emission line - which is present during high-luminosity spin- up periods - disappears when the luminosity drops, as the source goes into a spin-down epoch. It was further argued that this behavior was due to a geometric effect, the result of change in the emission diagram of the accretion column, which in turn is luminosity dependent (see e.g. Fig.1 of Schönherr et al. 2007, and references therein).

In this work we revisited the XMM-Newton observation for spectral analysis during the spin-down period and the recent $\mathrm{NuS}$ $T A R$ observation taken during the current spin-up phase. We note that our use of a MCD component for the modelling of the soft thermal emission does not necessarily signify emission from the inner part of an accretion disk heated due to viscous dissipation of gravitational energy. Indeed, the best fit value of the inner disk radius for the MCD model is of the order of $10-80 \mathrm{~km}$ for the two epoch; more than two orders of magnitude smaller than the expected size of the NS magnetosphere for the strength of its magnetic field and the source luminosity (assuming 20\% efficiency; see also estimations in Koliopanos \& Gilfanov 2016). It is very likely that the thermal component originates in optically thick material in the vicinity of the magnetosphere, which is further heated due to illumination from the central source emission - a phenomenon that is often detected in X-ray pulsars (see Endo et al. 2000 and Hickox et al. 2004 for a detailed description of this configuration and Koliopanos \& Vasilopoulos 2018 for a recent observational example).

Based on this scheme, the reasoning (that was also put forward in Koliopanos \& Gilfanov 2016) is that the disappearance of the iron line during the low-luminosity era, is due to significantly reduced illumination of the optically thick magnetospheric material, which in turn is the result of the changing beam pattern. This is further highlighted by the less luminous and significantly cooler soft emission, during the low-luminosity XMM-Newton observation (Table 1). The evolution to a fan-beam pattern emission, results in full illumination and heating of the optically thick material, and the appearance of the iron line, during the high luminosity phase, as observed with NUSTAR. We ran our code for a beam with a $20^{\circ}$ opening angle aimed at an optically thick rectangular slab using realistic abundances for a C/O WD donor, predicted by Gil-Pons \& García-Berro (2001), and the atomic cross-sections of Verner et al. (1996). If the material is illuminated in a face-on configuration we can reproduce an emission line with $\mathrm{EW}$ of $\sim 25 \mathrm{eV}$, for an incident spectrum with $\Gamma=0.7$, for the $\mathrm{C} / \mathrm{O}$-rich material. Increasing the inclination angle between the incident beam and the slab - thus reducing the reflected fraction - results in the complete disappearance of the emission line. Based on this analysis, one can argue that $4 \mathrm{U}$ 1626-67 has a C/O-rich donor. Nevertheless, the premise of He-rich donor and iron line variability that is entirely due to the geometrical configuration of the source, cannot be ruled out.

\subsection{Type I X-ray bursts and optical spectroscopy}

We made an effort to comprehensively report the bursting activity of all sources in the UCXB catalog, presented in Table 3. The recurrence rate of type I X-ray bursts can also indicate the chemical composition of the accreted material. Frequent bursts can be attributed to accretion of helium dominated material and/or residual hydrogen. On the other hand, sparse or no bursts - particularly in persistent sources - can be considered indicative of $\mathrm{C} / \mathrm{O}$ rich material with only limited amount of residual helium and/or hydrogen. In addition to their regularity, the characteristics of thermonuclear X-ray bursts - predominantly their duration and lightcurve shape - are heavily dependent on the chemical composition of the accumulated material which in turn affects the nuclear burn- 
ing. For instance, hydrogen burns to helium via the $\mathrm{CNO}$ cycle and helium burns to carbon through the triple-alpha process. In broad terms, type I X-ray bursts can be divided into three categories based on their duration. Short bursts $(\sim 10-100 \mathrm{~s}$, associated with surface, unstable $\mathrm{H}$ and He burning, see reviews by Lewin et al. 1993 and Strohmayer \& Bildsten 2006), intermediate bursts ( 15-40 m, most likely powered by the burning of a thick layer of helium, e.g. Cumming et al. 2006) and the so-called superbursts ( 1 day, powered by carbon burning, e.g. Keek et al. 2008).

All sources in the UCXB catalog - for which no iron line is detected in their spectra (including the variable sources) - exhibit sparse type I X-ray bursts or no bursts at all. The source with most bursts detected is $4 \mathrm{U}$ 0513-40 (35 bursts detected in over a decade of monitoring). Indeed, $4 \mathrm{U} 0513-40$ is Out of the 10 sources with no iron emission features, six are persistent sources (4U 051340, 2S 0918-549, 4U 1543-624, 4U 1850-087, 4U 2129+11 and 4 U 1626-67) and four are transient (XTE J0929-314, XTE J1751305, Swift J1756.9-2508, XTE J1807-294). On the other hand, the five out of six UCXBs with prominent and persistent iron emission lines are frequent bursters with hundreds of type I X-ray bursts recorded and some with estimated recurrence rates in the order of days or hours. Four of these five sources, are persistent emitters (4U 0614+091, 4U 1728-34, 4U 1820-30, 4U 1916-05), while the fifth (IGR J17062-6143) is a transient source which has produced type I X-ray bursts during both of its outbursts. The sixth UCXB with an iron line detection is NGC $6440 \mathrm{X}-2$, which is a transient source. No bursts have been observed from this source, but it is important to note that NGC 6440 X-2 exhibits faint outbursts with very short duration, that usually go undetected by all-sky monitors (Altamirano et al. 2010), for this reason there are only few available observations for this source.

In addition to their high recurrence rates, the burst characteristics of sources 4U 1820-30 (e.g. Bildsten 1995; Cumming 2003; Galloway et al. 2008), 4U 1916-05 (e.g. Galloway et al. 2008), 4 U 1728-34 (e.g. Galloway et al. 2008; Misanovic et al. 2010), 4U 0614+091 (e.g. Linares et al. 2012) and IGR J17062-6143 (e.g. Keek et al. 2017) are also consistent with helium burning. Most UCXB companion stars and accretion disks are too faint for optical spectroscopy. 4U 1916-05 has optical confirmation of a He-rich donor (Nelemans et al. 2006), while tentative optical evidence for a C/O companion have been put forward for 4U 1626-67, 2S 0918549 (orbital period and low mass-transfer rate are also consistent with low-entropy C/O white dwarf donor, Heinke et al. 2013) and 4U 1543-624 (Nelemans et al. 2004; Werner et al. 2006) and FUV for $4 \mathrm{U}+2129+11$ ( $\mathrm{C}$ and He lines Dieball et al. 2005). On the other hand, contrary to our designation as a He-rich source, Nelemans et al. (2004) and Werner et al. (2006) have proposed a C/O-rich donor for 4U 0614+091, based on the presence of $\mathrm{C}$ and $\mathrm{O}$ emission lines on optical spectrum from the VLT. Our re-classification, however, not only handily explains its persistent bursting activity, but is also in agreement with indications for a He-rich donor based on arguments on disk stability and evolution (Heinke et al. 2013). FUV (Homer et al. 2002) and optical (Werner et al. 2006) spectroscopy of $4 \mathrm{U}$ 1626-67 supports the presence of C/O-rich donor, in agreement to our classification. However, Heinke et al. (2013) argue that the orbital period and persistent accretion of the source can best be explained by He-star models rather than a C/O-rich donor.

\subsection{X-ray spectroscopy in the bibliography}

Meticulous scrutiny of the X-ray spectral continuum of $4 \mathrm{U}$ 051340, 4U 0614+091, 2S 0918-549 and 4U 1850-087 - using moder- ate (ASCA, BeppoSAX and XMM-Newton EPIC) and high resolution instruments (Chandra and XMM-Newton grating spectrometers) - have revealed the presence of unusually strong absorption edges, indicating highly non-solar composition of the local interstellar medium, attributed to $\mathrm{C} / \mathrm{O}$ or $\mathrm{O} / \mathrm{Ne} / \mathrm{Mg}$-rich donors (e.g. Paerels et al. 2001; Juett et al. 2001; Juett \& Chakrabarty 2003). However, analysis of later observations, yielded standard solar-like abundances, revealing a temporal variability of the effect and thus indicating that the apparent non-solar abundances could be the result of fluctuations in the ionization state of the surrounding material (e.g. Juett \& Chakrabarty 2005; Schulz et al. 2010), rather than the result of non-solar composition. Low-energy $(\lessgtr 1 \mathrm{keV})$ broad emission-like features have also been detected in sources 4U 0614+091 (Madej et al. 2010), 4U 1453-624 (Madej \& Jonker 2011) and 4U 1626-67 Krauss et al. (2007) and have been attributed to emission from overabundant carbon, oxygen and/or neon in the accretion disk material (e.g. Madej et al. 2014).

An interesting point is raised by the modelling of 4U 0614+091 by Madej et al. (2014). In this work, they use a version of the XILLVER X-ray reflection code (García \& Kallman 2010; García et al. 2011; García et al. 2013) modified in order to simulate reflection by $\mathrm{C} / \mathrm{O}$-rich material (the authors refer to this version of their code as XILLVER ${ }_{\mathrm{CO}}$ ). They successfully model the soft emission-like features as well as the iron $\mathrm{K} \alpha$ emission line and argue that the soft features are due to a C/O-rich accretion disk. This finding seemingly contradicts the findings of our own MC simulations which clearly indicate the screening of the iron line (primarily) by the overabundant oxygen. However, it is important to

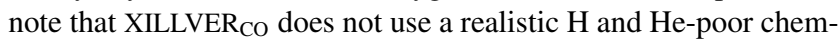
ical composition, but rather simulates it by artificially increasing the abundance of all elements apart from $\mathrm{H}, \mathrm{He}, \mathrm{C}$ and $\mathrm{O}$ by $\times 10$ times and then further increases the abundances of $\mathrm{C}$ and $\mathrm{O}$ producing a grid of abundance patterns in order to fit the spectra of $4 \mathrm{U} 0614+091$. In the two observations where their fit constrains the $\mathrm{C} / \mathrm{O}$ abundance, they report a value ranging between 130 to $160 \times$ their solar value. However, based on their scheme for mimicking the $\mathrm{C} / \mathrm{O}$-rich disk, their actual $\mathrm{O} / \mathrm{Fe}$ ratio is $13-16 \times$ its solar value; at least five times lower than the $\mathrm{O} / \mathrm{Fe}$ ratio of an actual $\mathrm{H}$-poor, C/O-rich disk (i.e. 80, Koliopanos et al. 2013; Koliopanos et al. 2014 and this work). Furthermore, the accretion disk simulated by XILLVER $_{\text {CO }}$ in Madej et al. is still largely dominated by hydrogen and helium, rather than carbon and oxygen - which significantly affects the ionization state of the disk. On the other hand, while our own simulation features a realistic disk composition, it does not self-consistently estimate the ionization state of the C/O-rich disk. A combination of the merits of our own simulations and the highly sophisticated model of García \& Kallman will be attempted in a future project.

Lastly, we note that in this work we have not included the notable UCXB candidate 47 Tuc X9, whose observed radio/X-ray luminosity ratio is consistent with a black hole accretor (Miller-Jones et al. 2015) and $\sim 28$ min periodic modulation of its X-ray emission strongly indicates an orbital period consistent with UCXBs. Subsequent, extensive analysis of the rich archive of X-ray observations of 47 Tuc X9 provided further evidence strongly favoring the scenario that this is the first black hole UCXB (Bahramian et al. 2017). Spectral and temporal analysis of the X-ray emission by Bahramian et al. indicates a C/O-rich WD donor star. The detection of strong oxygen lines below $1 \mathrm{keV}$ and the absence of an iron emission line, is in agreement with our predictions for $\mathrm{C} / \mathrm{O}$-rich donors. The absence of any type I X-ray bursts from this source is also consistent with our predictions (but it is also consistent with the black 
hole accretor, regardless of the chemical composition of the donor material). However, the low source luminosity $\left(L_{X}<10^{34} \mathrm{erg} / \mathrm{s}\right)$ suggests a very low-accretion state that points to the presence of an optically thin inner accretion flow (with possible optically thin plasma emission) and an accretion disk that is truncated at a distance that is at least an order of magnitude larger than in our Monte Carlo simulation. Therefore, we would need to make significant modifications to our code, in order to make robust claims about the nature of 47 Tuc X9. This is beyond the scope of this paper and is considered for a separate publication.

\section{CONCLUSIONS}

The analysis of the X-ray spectra of all known UCXBs - using the predictions of the X-ray reflection code developed in Koliopanos et al. (2013) - has enabled us to draw potential conclusions for the chemical composition of their accretion disks and donor star. We found that all UCXBs featuring a prominent iron $\mathrm{K} \alpha$ emission line in their X-ray spectra, that is persistent over multiple observations, also exhibit a rich and recurrent bursting activity. On the other hand, all UCXBs for which the iron line is not detected in all or most of their available spectra, also have few or no type I X-ray bursts detected, in over more than a decade of monitoring. Based on the strong correlation between the strength of the $\mathrm{Fe} \mathrm{K} \alpha$ emission line and the abundance of carbon and oxygen in the accretion disk material - demonstrated in Koliopanos et al. (2013) - and the correlation between the abundance of hydrogen and helium in the recurrence rate of short and intermediate type I X-ray bursts, we argue that there is a strong likelihood that all known UCXBs with persistent iron emission have He-rich donors, while those that do not, likely have $\mathrm{C} / \mathrm{O}$ or $\mathrm{O} / \mathrm{Ne} / \mathrm{Mg}$-rich donors.

If confirmed, this result can have profound implications in our understanding of compact binary evolution especially since standard population synthesis models tend to produce an overwhelming majority of He-rich systems rather than C/O-rich systems (e.g. Nelemans et al. 2010). Furthermore, recent hydrodynamic simulations of mass transfer in binaries indicate that NS's with $\mathrm{CO}$ or $\mathrm{O} / \mathrm{Ne}$ WD donors lead to rapid NS-WD mergers and thus UCXBs with such donors would be very short-lived systems (Bobrick et al. 2017). Comparison of our conclusions with assessments based on optical and X-ray spectroscopy returned positive results, but also some tensions. We also found indications of the iron line variability in C/O-rich disks, predicted in Koliopanos et al. (2013). Overall,it becomes evident that an increase in the sophistication of our $\mathrm{X}$-ray reflection models is required concerning the problem of nonsolar abundances and disk ionization in order to definitively confirm our current conclusions. We therefore only regard the results of this work as encouraging and intriguing and conclude that they certainly justify further exploration.

\section{ACKNOWLEDGEMENTS}

F.K. is deeply indebted to Marat Gilfanov, whose guidance made this series of projects possible. F.K. is also grateful for the collaboration of Lars Bildsten and Maria Diaz Trigo, in the works that preceded and led to this final project. Lastly, F.K. extends his utmost gratitude to the referee and editor of this paper, for their contribution to its final form, but more importantly their patience during a prolonged pause in the refereeing process, caused by F.K., due to an unforeseen personal issue.

\section{DATA AVAILABILITY}

The observational data underlying this article are publicly available at the NASA HEASARC archive ${ }^{11}$. Any details with regard to the Monte Carlo source code can be shared on reasonable request to the corresponding author.

\section{REFERENCES}

Altamirano D., et al., 2010, ApJ, 712, L58

Amaro-Seoane P., et al., 2017, arXiv e-prints, p. arXiv:1702.00786

Anders E., Grevesse N., 1989, Geochimica Cosmochimica Acta, 53, 197

Arnaud K. A., 1996, in Jacoby G. H., Barnes J., eds, Astronomical Society of the Pacific Conference Series Vol. 101, Astronomical Data Analysis Software and Systems V. p. 17

Bahramian A., et al., 2017, MNRAS, 467, 2199

Ballantyne D. R., Ross R. R., Fabian A. C., 2001, MNRAS, 327, 10

Bambynek W., Crasemann B., Fink R. W., Freund H.-U., Mark H., Swift C. D., Price R. E., Rao P. V., 1972, Reviews of Modern Physics, 44, 716

Barret D., 2001, Advances in Space Research, 28, 307

Basko M. M., Sunyaev R. A., Titarchuk L. G., 1974, A\&A, 31, 249

Belczynski K., Taam R. E., 2004, ApJ, 603, 690

Bildsten L., 1995, ApJ, 438, 852

Bildsten L., Deloye C. J., 2004, ApJ, 607, L119

Bobrick A., Davies M. B., Church R. P., 2017, MNRAS, 467, 3556

Boirin L., Parmar A. N., Barret D., Paltani S., Grindlay J. E., 2004, A\&A, 418, 1061

Brandt S., Castro-Tirado A. J., Lund N., Dremin V., Lapshov I., Syunyaev R., 1992, A\&A, 262, L15

Cackett E. M., et al., 2010, ApJ, 720, 205

Chakrabarty D., 1998, ApJ, 492, 342

Chakrabarty D., Morgan E. H., 1998, Nature, 394, 346

Cornish N., Robson T., 2017, in Journal of Physics Conference Series. p. 012024 (arXiv: 1703.09858), doi:10.1088/1742-6596/840/1/012024

Cumming A., 2003, ApJ, 595, 1077

Cumming A., Macbeth J., in 't Zand J. J. M., Page D., 2006, ApJ, 646, 429

Degenaar N., Miller J. M., Wijnands R., Altamirano D., Fabian A. C., 2013, ApJ, 767, L37

Deloye C. J., Bildsten L., 2003, ApJ, 598, 1217

Deloye C. J., Bildsten L., Nelemans G., 2005, ApJ, 624, 934

Dieball A., Knigge C., Zurek D. R., Shara M. M., Long K. S., Charles P. A., Hannikainen D. C., van Zyl L., 2005, ApJ, 634, L105

Done C., Gierliński M., Kubota A., 2007, A\&ARv, 15, 1

Endo T., Nagase F., Mihara T., 2000, PASJ, 52, 223

Fabian A. C., Rees M. J., Stella L., White N. E., 1989, MNRAS, 238, 729

Fiocchi M., Bazzano A., Natalucci L., Landi R., Ubertini P., 2011, MNRAS, 414, L41

Galloway D. K., 2006, in D'Amico F., Braga J., Rothschild R. E., eds, American Institute of Physics Conference Series Vol. 840, The Transient Milky Way: A Perspective for MIRAX. pp 50-54 (arXiv: astro-ph/0604345), doi:10.1063/1.2216602

Galloway D. K., Chakrabarty D., Morgan E. H., Remillard R. A., 2002, ApJ, 576, L137

Galloway D. K., Muno M. P., Hartman J. M., Psaltis D., Chakrabarty D., 2008, ApJS, 179, 360

Galloway D. K., Yao Y., Marshall H., Misanovic Z., Weinberg N., 2010, The Astrophysical Journal, 724, 417

García J., Kallman T. R., 2010, ApJ, 718, 695

García J., Kallman T. R., Mushotzky R. F., 2011, ApJ, 731, 131

García J., Dauser T., Reynolds C. S., Kallman T. R., McClintock J. E., Wilms J., Eikmann W., 2013, ApJ, 768, 146

George I. M., Fabian A. C., 1991, MNRAS, 249, 352

Gil-Pons P., García-Berro E., 2001, A\&A, 375, 87

11 https://heasarc.gsfc.nasa.gov/docs/archive.html 
Gilfanov M., 2010, in Belloni T., ed., Lecture Notes in Physics, Berlin Springer Verlag Vol. 794, Lecture Notes in Physics, Berlin Springer Verlag. p. 17, doi:10.1007/978-3-540-76937-8_2

Grindlay J., Gursky H., Schnopper H., Parsignault D. R., Heise J., Brinkman A. C., Schrijver J., 1976, ApJ, 205, L127

HI4PI Collaboration et al., 2016, A\&A, 594, A116

Hansen C. J., van Horn H. M., 1975, ApJ, 195, 735

Harris W. E., 2010, arXiv e-prints, p. arXiv:1012.3224

Heinke C. O., Ivanova N., Engel M. C., Pavlovskii K., Sivakoff G. R., Cartwright T. F., Gladstone J. C., 2013, ApJ, 768, 184

Hickox R. C., Narayan R., Kallman T. R., 2004, ApJ, 614, 881

Homer L., Charles P. A., Naylor T., van Paradijs J., Auriere M., KochMiramond L., 1996, MNRAS, 282, L37

Homer L., Anderson S. F., Wachter S., Margon B., 2002, AJ, 124, 3348

Iben Jr. I., Tutukov A. V., Yungelson L. R., 1995, ApJS, 100, 233

Iwakiri W. B., et al., 2019, ApJ, 878, 121

Jahoda K., Markwardt C. B., Radeva Y., Rots A. H., Stark M. J., Swank J. H., Strohmayer T. E., Zhang W., 2006, ApJS, 163, 401

Jenke P. A., Linares M., Connaughton V., Beklen E., Camero-Arranz A., Finger M. H., Wilson-Hodge C. A., 2016, ApJ, 826, 228

Juett A. M., Chakrabarty D., 2003, ApJ, 599, 498

Juett A. M., Chakrabarty D., 2005, ApJ, 627, 926

Juett A. M., Chakrabarty D., 2006, ApJ, 646, 493

Juett A. M., Psaltis D., Chakrabarty D., 2001, ApJ, 560, L59

Keek L., in't Zand J. J. M., Kuulkers E., Cumming A., Brown E. F., Suzuki M., 2008, A\&A, 479, 177

Keek L., Iwakiri W., Serino M., Ballantyne D. R., in’t Zand J. J. M., Strohmayer T. E., 2017, ApJ, 836, 111

Koliopanos F., Gilfanov M., 2016, MNRAS, 456, 3535

Koliopanos F., Vasilopoulos G., 2018, A\&A, 614, A23

Koliopanos F., Gilfanov M., Bildsten L., 2013, MNRAS, 432, 1264

Koliopanos F., Gilfanov M., Bildsten L., Trigo M. D., 2014, MNRAS, 442, 2817

Krauss M. I., Schulz N. S., Chakrabarty D., Juett A. M., Cottam J., 2007, ApJ, 660, 605

Krimm H. A., et al., 2007, ApJ, 668, L147

Lewin W. H. G., van Paradijs J., Taam R. E., 1993, Space Sci. Rev., 62, 223

Lightman A. P., White T. R., 1988, ApJ, 335, 57

Lin D., Remillard R. A., Homan J., 2007, ApJ, 667, 1073

Linares M., et al., 2012, ApJ, 760, 133

Madej O. K., Jonker P. G., 2011, MNRAS, 412, L11

Madej O. K., Jonker P. G., Fabian A. C., Pinto C., Verbunt F., de Plaa J., 2010, MNRAS, 407, L11

Madej O. K., García J., Jonker P. G., Parker M. L., Ross R., Fabian A. C., Chenevez J., 2014, MNRAS, 442, 1157

Markwardt C. B., Swank J. H., Strohmayer T. E., in 't Zand J. J. M., Marshall F. E., 2002, ApJ, 575, L21

Markwardt C. B., Juda M., Swank J. H., 2003, The Astronomer's Telegram, 127,1

Matt G., Fabian A. C., Ross R. R., 1993, MNRAS, 262, 179

Matt G., Fabian A. C., Reynolds C. S., 1997, MNRAS, 289, 175

Miller-Jones J. C. A., et al., 2015, MNRAS, 453, 3918

Misanovic Z., Galloway D. K., Cooper R. L., 2010, ApJ, 718, 947

Nelemans G., Jonker P. G., 2010, New Astron. Rev., 54, 87

Nelemans G., Jonker P. G., Marsh T. R., van der Klis M., 2004, MNRAS, 348, L7

Nelemans G., Jonker P. G., Steeghs D., 2006, MNRAS, 370, 255

Nelemans G., Yungelson L. R., van der Sluys M. V., Tout C. A., 2010, MNRAS, 401, 1347

Nelson L. A., Rappaport S. A., Joss P. C., 1986a, ApJ, 304, 231

Nelson L. A., Rappaport S. A., Joss P. C., 1986b, ApJ, 311, 226

Ng C., Díaz Trigo M., Cadolle Bel M., Migliari S., 2010, A\&A, 522, A96

Ortolani S., Barbuy B., Bica E., 1994, A\&AS, 108, 653

Paerels F., et al., 2001, ApJ, 546, 338

Papitto A., Menna M. T., Burderi L., di Salvo T., Riggio A., 2008, MNRAS, 383,411

Patruno A., Altamirano D., Messenger C., 2010, MNRAS, 403, 1426

Podsiadlowski P., Rappaport S., Pfahl E. D., 2002, ApJ, 565, 1107

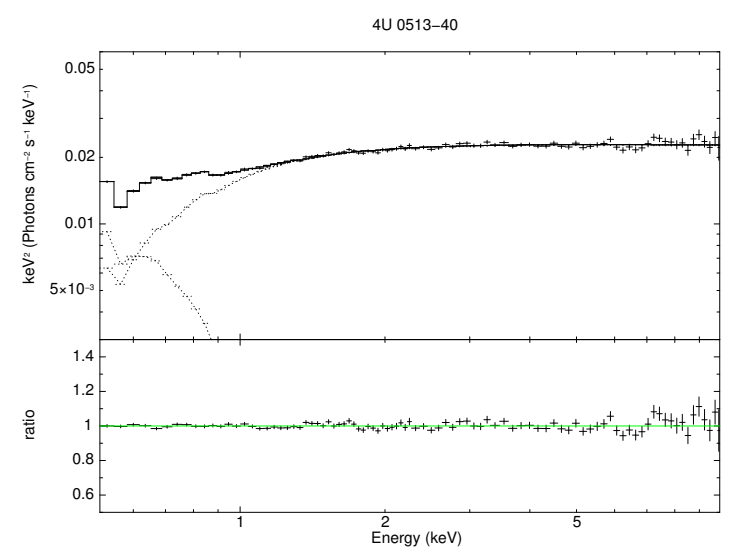

Figure A1. 4U 0513-40: Unfolded spectrum. Energy and data-vs-model ratio plot, for only the continuum model.

Popham R., Sunyaev R., 2001, ApJ, 547, 355

Pringle J. E., Webbink R. F., 1975, MNRAS, 172, 493

Rappaport S., Joss P. C., 1984, ApJ, 283, 232

Savonije G. J., de Kool M., van den Heuvel E. P. J., 1986, A\&A, 155, 51

Schönherr G., Wilms J., Kretschmar P., Kreykenbohm I., Santangelo A., Rothschild R. E., Coburn W., Staubert R., 2007, A\&A, 472, 353

Schultz J., 2003, A\&A, 397, 249

Schulz N. S., Nowak M. A., Chakrabarty D., Canizares C. R., 2010, ApJ, 725,2417

Shahbaz T., Watson C. A., Zurita C., Villaver E., Hernandez-Peralta H., 2008, PASP, 120, 848

Stella L., Priedhorsky W., White N. E., 1987, ApJ, 312, L17

Strohmayer T., Bildsten L., 2006, New views of thermonuclear bursts. Cambridge University Press, pp 113-156

Strohmayer T. E., et al., 2018, ApJ, 858, L13

Tutukov A. V., Yungelson L. R., 1993, Astronomy Reports, 37, 411

Verbunt F., van den Heuvel E. P. J., 1995, X-ray Binaries, pp 457-494

Verner D. A., Ferland G. J., Korista K. T., Yakovlev D. G., 1996, ApJ, 465, 487

Walter F. M., Mason K. O., Clarke J. T., Halpern J., Grindlay J. E., Bowyer S., Henry J. P., 1982, ApJ, 253, L67

Wang Z., Chakrabarty D., 2004, ApJ, 616, L139

Werner K., Nagel T., Rauch T., Hammer N. J., Dreizler S., 2006, A\&A, 450, 725

White T. R., Lightman A. P., Zdziarski A. A., 1988, ApJ, 331, 939

Wilms J., Allen A., McCray R., 2000, ApJ, 542, 914

Yungelson L. R., Nelemans G., van den Heuvel E. P. J., 2002, A\&A, 388, 546

Zhong J., Wang Z., 2011, ApJ, 729, 8

Zurek D. R., Knigge C., Maccarone T. J., Dieball A., Long K. S., 2009, ApJ, 699, 1113

in't Zand J. J. M., Cumming A., van der Sluys M. V., Verbunt F., Pols O. R., 2005, A\&A, 441, 675

van Paradijs J., McClintock J. E., 1994, A\&A, 290, 133

van Paradijs J., Dotani T., Tanaka Y., Tsuru T., 1990, PASJ, 42, 633

\section{APPENDIX A: SOME EXTRA MATERIAL}

This paper has been typeset from a $\mathrm{T}_{\mathrm{E}} \mathrm{X} / \mathrm{L} \mathrm{AT} \mathrm{E}$ file prepared by the author. 
Table A1. Best fit parameters for the continuum emission spectra of the three UCXBs with variable iron line emission. 4U 1626-67 - a high-B NS, X-ray pulsar - is tabulated separately. All errors are in the $1 \sigma$ confidence range.

\begin{tabular}{|c|c|c|c|c|c|c|c|c|c|}
\hline Source & $\begin{array}{c}\mathrm{nH} \\
\times 10^{22}\end{array}$ & $\begin{array}{c}\mathrm{kT}_{\mathrm{dBB}} \\
\mathrm{keV}\end{array}$ & $\begin{array}{c}\mathrm{R}_{\mathrm{dBB}^{a}} \\
\mathrm{~km}\end{array}$ & $\Gamma$ & $\begin{array}{c}\mathrm{K}_{\mathrm{po}}^{b} \\
{\left[10^{-3}\right]}\end{array}$ & $\mathrm{kT}_{\mathrm{BB}}$ & $\begin{array}{c}\mathrm{R}_{\mathrm{BB}}{ }^{c} \\
\mathrm{~km}\end{array}$ & $\begin{array}{c}\mathrm{L}^{\mathrm{d}} \\
10^{36} \times \mathrm{erg} \mathrm{s}^{-1}\end{array}$ & red. $\chi^{2} / d o f$ \\
\hline $4 \mathrm{U} 1543-624^{h}(1997)$ & $0.29^{f}$ & $0.59_{-0.22}^{+0.31}$ & $11.5_{-1.56}^{+7.06^{g}}$ & $4.32_{-1.22}^{+1.31}$ & $0.35 \pm 0.05$ & $1.53 \pm 0.01$ & $0.32 \pm 0.06$ & 6.64 & $0.64 / 38$ \\
\hline Swift J1756.9-2508 (2007) & $0.01^{f}$ & $1.85_{-0.28}^{+0.26}$ & $6.2_{-1.35}^{+3.76}$ & - & - & $4.15_{-1.01}^{+0.82}$ & $0.10 \pm 0.05$ & 4.53 & $0.62 / 32$ \\
\hline 4U 1626-67 (2015) & $1.00^{f}$ & $0.68_{-0.22}^{+0.31}$ & $9.72_{-1.22}^{+1.31}$ & $1.02 \pm 0.004$ & $30.6_{-2.8}^{+3.2}$ & - & - & 3.04 & $1.05 / 838$ \\
\hline
\end{tabular}

${ }^{a} R_{\mathrm{dBB}}$ (the inner radius of the accretion disk in $\mathrm{km}$ ) is inferred from diskbb model, by solving $\mathrm{K}_{\mathrm{dBB}}=\left(\mathrm{R}_{\mathrm{dBB}} / \mathrm{D}_{10}\right)^{2} \cos i$, for $R_{\mathrm{dBB}}$. ' $\mathrm{K}_{\mathrm{BB}}$ ' is the normalisation of the diskbb model, $\mathrm{D}_{10}$ is distance in units of $10 \mathrm{kpc}$ and $i$ is the inclination. In all sources this was assumed to be $60^{\circ}$, with the exceptions of 4U 1916-05 and 4U 0513-40 (where $i$ is set to $85^{\circ}$ ), which are known edge-on viewed systems (Boirin et al. 2004; Fiocchi et al. 2011). With the exception of XTE J19029-314 (for which the K estimation has considerably high $1 \sigma$ error bars), errors in radius estimation are dominated by the $1 \sigma$ errors in the distance estimation (see Table 1).

${ }^{b}$ Power-law component normalisation constant: photons $/ \mathrm{keV} / \mathrm{cm}^{2} / \mathrm{s}$ at $1 \mathrm{keV}$

${ }^{c}$ Size of the spherical black body component (bbodyrad model), estimated from $\mathrm{K}_{\mathrm{BB}}=\mathrm{R}_{\mathrm{BB}}{ }^{2} / \mathrm{D}_{10}{ }^{2}$, where $\mathrm{K}_{\mathrm{BB}}$ is the normalization parameter of the bbodyrad model.

${ }^{d}$ Luminosity in the $0.5-60 \mathrm{keV}$ range, extrapolated from the best-fit model.

${ }^{e}$ Emission and/or absorption-like features detected $<1 \mathrm{keV}$ were modelled using a combination of Gaussian absorption lines. For justification and details see Section 3.4 .

${ }^{f}$ Parameter frozen at total galactic H I column density (HI4PI Collaboration et al. 2016).

${ }^{g}$ There is considerably uncertainty in the distance estimation for these two sources. Radius is estimated for a distance of $7 \mathrm{kpc}$ (Wang \& Chakrabarty 2004) for $4 \mathrm{U} 1543-624$ and $8 \mathrm{kpc}$ for $4 \mathrm{U}$ 1626-67 (4-13 kpc; Chakrabarty \& Morgan 1998). Error bars are estimated from the $1 \sigma$ errors for the best-fit value of $K_{\mathrm{dBB}}$.

${ }^{h}$ With an additional "constrained" broken power law ( $p_{1} \leq 2.5$ and $\left.E_{b}=20 \mathrm{keV}\right)$. This empirical model for high energy tails of NS XRBs in soft state, is often used in broadband X-ray spectroscopy (see Lin et al. 2007 and Koliopanos et al. submitted for more details). In the table we tabulate the value of $p_{2}$ for the spectral index value.

Table A2. Best fit parameters for iron line emission in the three UCXBs with variable line detection. 4U 1626-67 - a high-B NS, X-ray pulsar - is tabulated separately. The errors are $1 \sigma$.

\begin{tabular}{lccc}
\hline Model parameter & 4U 1543-624 (1997) & Swift J1756.9-2508 (2007) & 4U 1626-67 (2010) \\
\hline Iron Line & & & \\
\hline Centroid E (keV) & $6.58_{-0.33}^{+0.21}$ & $6.58 \pm 0.06$ & $6.76 \pm 0.03$ \\
Width $\sigma(\mathrm{keV})$ & $0.68_{-0.36}^{+0.55}$ & $0.1^{b}$ & $0.19 \pm 0.04$ \\
Flux $^{a}$ & $95.1_{-2.5}^{+34.5}$ & $42.6 \pm 4.60$ & $13.4_{-1.61}^{+1.69}$ \\
EW $(\mathrm{eV})$ & $118_{-24.3}^{+66.6}$ & $173 \pm 18.5$ & $30.1 \pm 3.85$ \\
\hline
\end{tabular}

${ }^{a} 10^{-5} \mathrm{ph} \mathrm{cm}^{-2} \mathrm{~s}^{-1}$.

${ }^{b}$ Parameter frozen at minimum spectral resolution value.

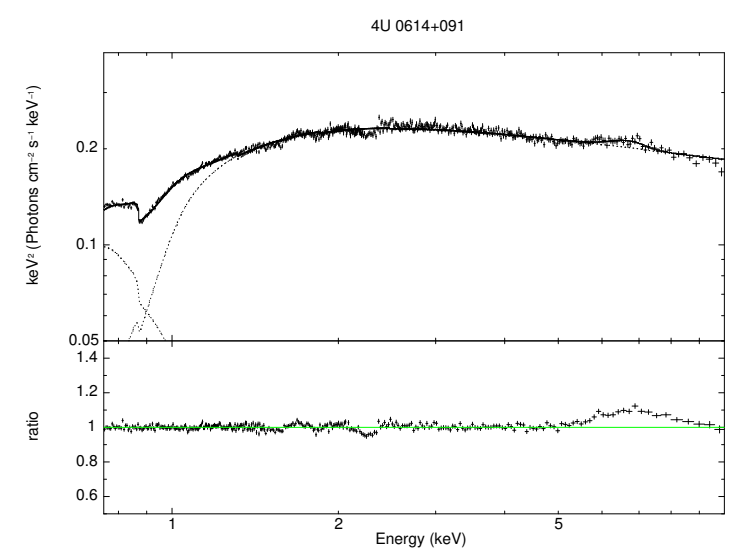

Figure A2. 4U 0614+091: Unfolded spectrum. Energy and data-vs-model ratio plot, for only the continuum model.

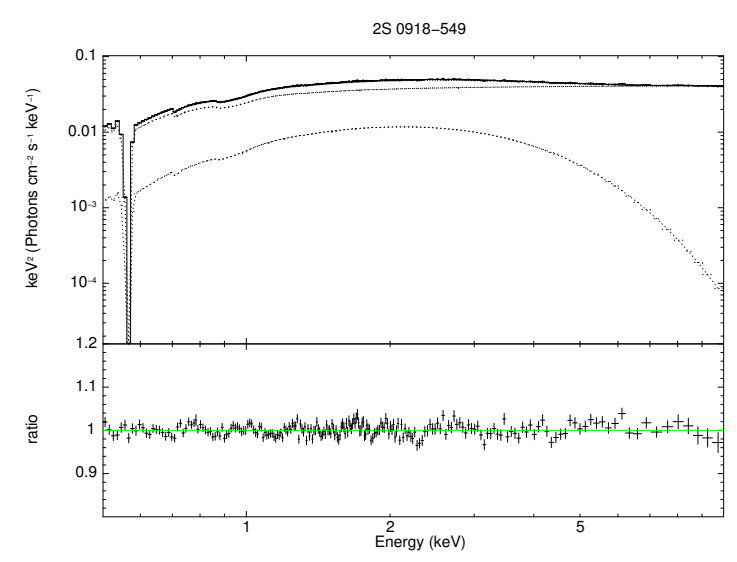

Figure A3. 2 S 0918-549: Unfolded spectrum. Energy and data-vs-model ratio plot, for only the continuum model. 


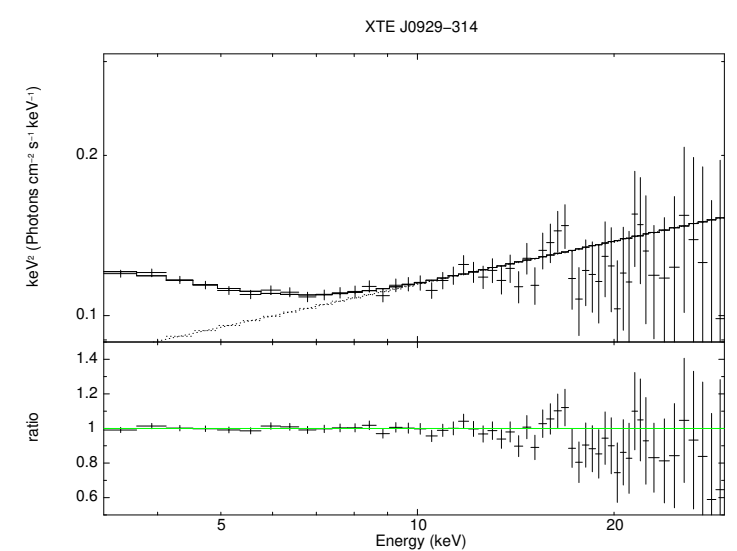

Figure A4. XTE J0929-314: Unfolded spectrum. Energy and data-vs-model ratio plot, for only the continuum model.

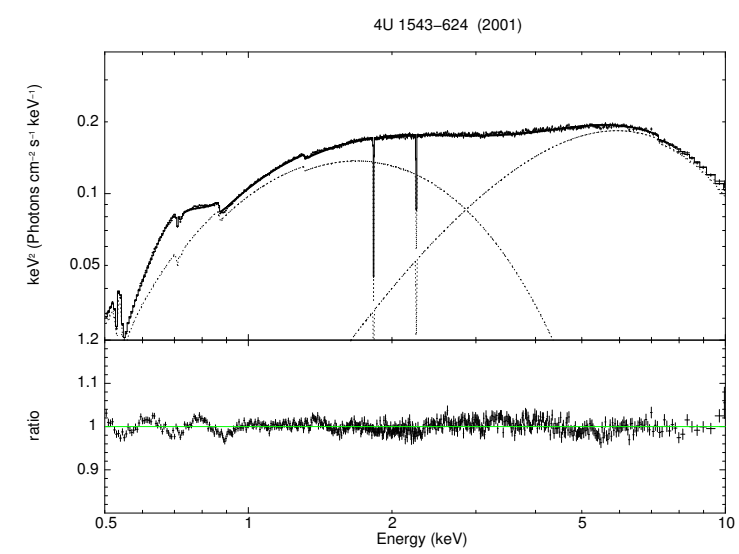

Figure A5. 4U 1543-624 (2001): Unfolded spectrum. Energy and data-vsmodel ratio plot, for only the continuum model.

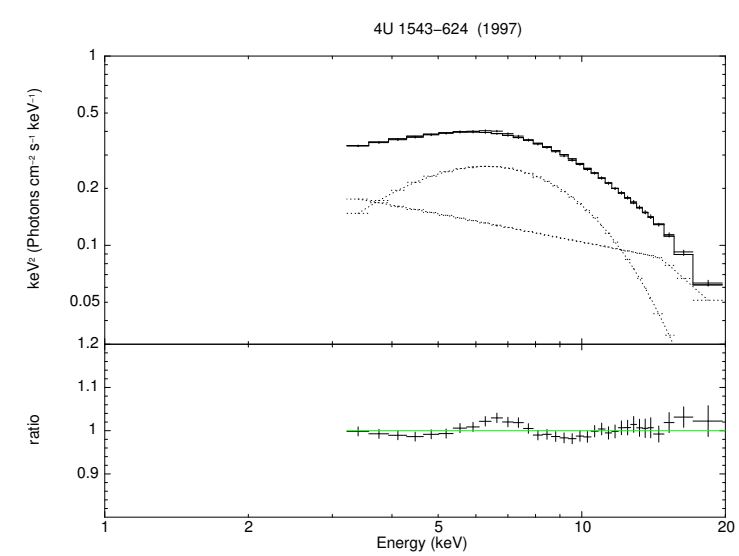

Figure A6. 4U 1543-624 (1997): Unfolded spectrum. Energy and data-vsmodel ratio plot, for only the continuum model.

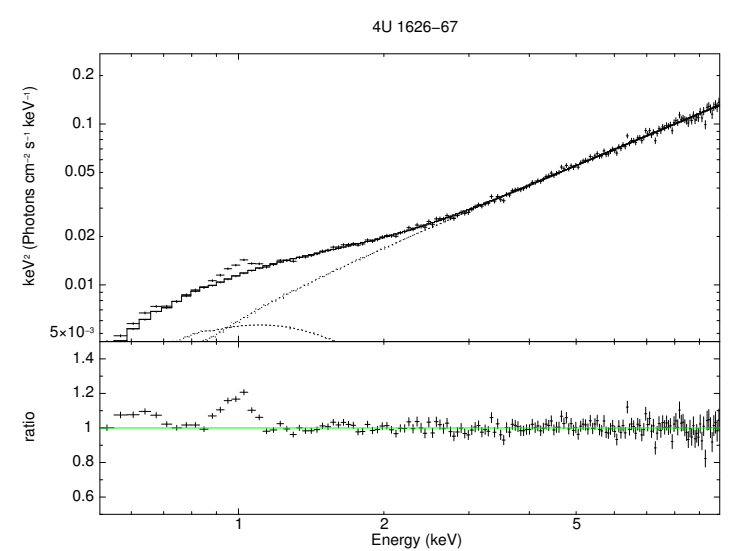

Figure A7. 4U 1626-67: Unfolded spectrum. Energy and data-vs-model ratio plot, for only the continuum model.

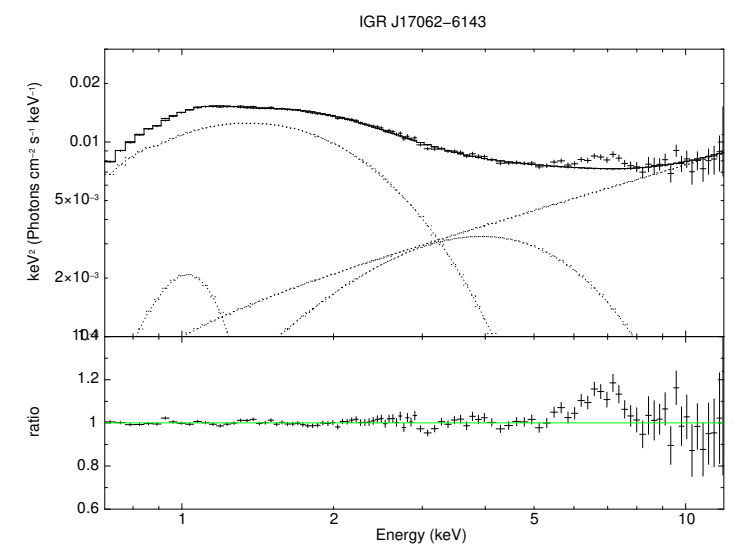

Figure A8. IGR J17062-6143: Unfolded spectrum. Energy and data-vsmodel ratio plot, for only the continuum model.

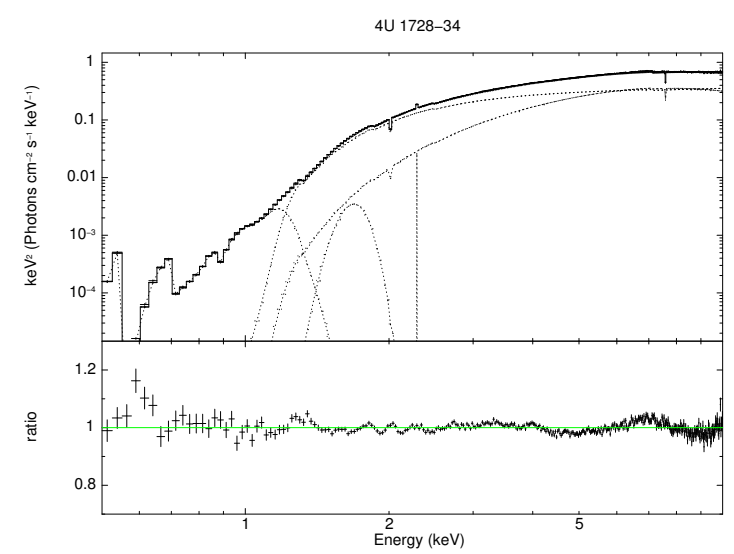

Figure A9. 4U 1728-34: Unfolded spectrum. Energy and data-vs-model ratio plot, for only the continuum model. 


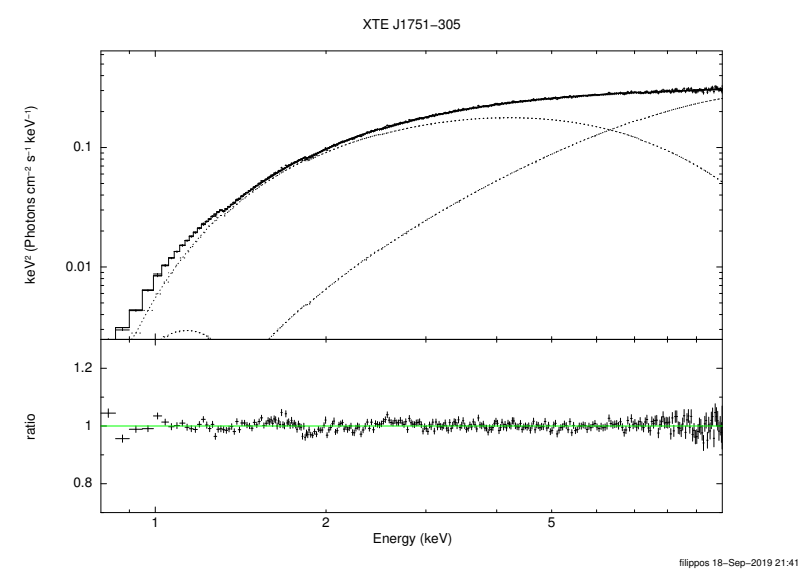

Figure A10. XTE J1751-305: Unfolded spectrum. Energy and data-vsmodel ratio plot, for only the continuum model.

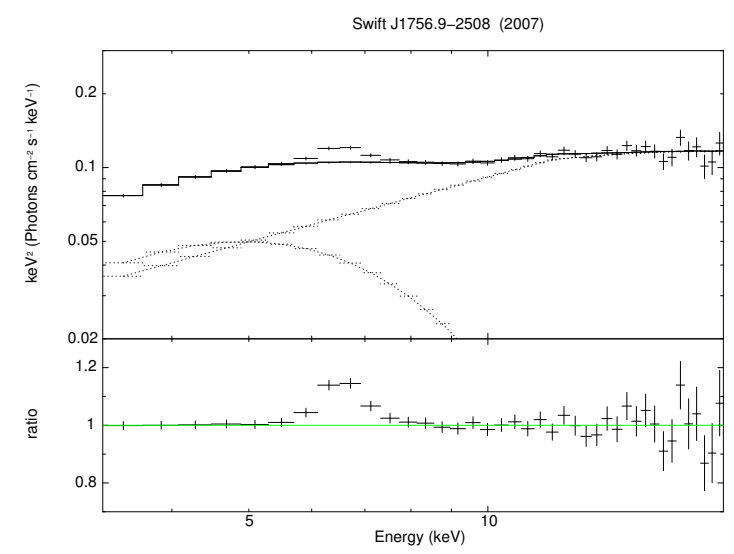

Figure A11. Swift J1756.9-2508 (2007): Unfolded spectrum. Energy and data-vs-model ratio plot, for only the continuum model.

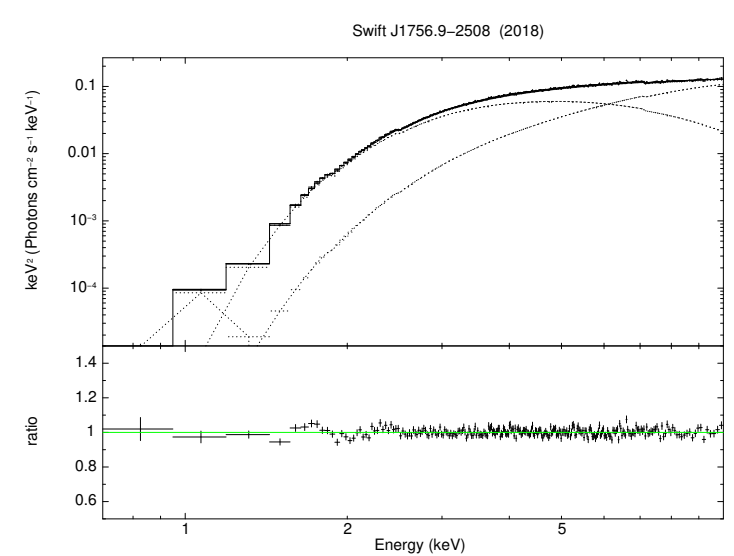

Figure A12. Swift J1756.9-2508 (2018): Unfolded spectrum. Energy and data-vs-model ratio plot, for only the continuum model.

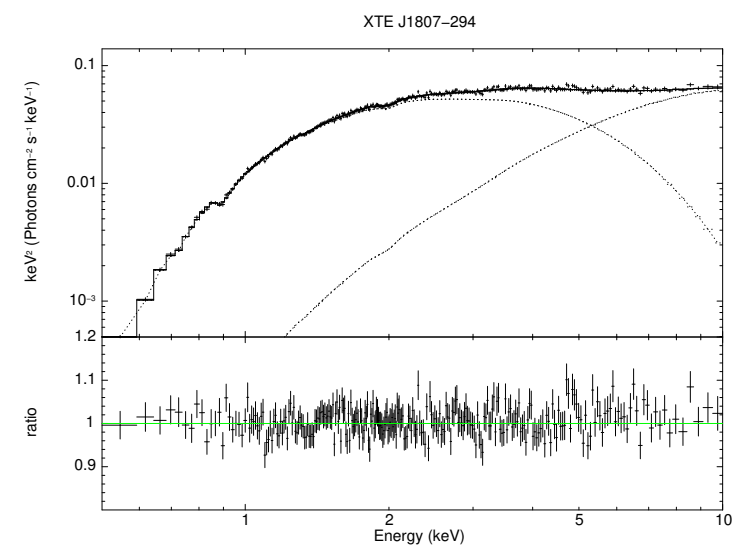

Figure A13. XTE J1807-294: Unfolded spectrum. Energy and data-vsmodel ratio plot, for only the continuum model.

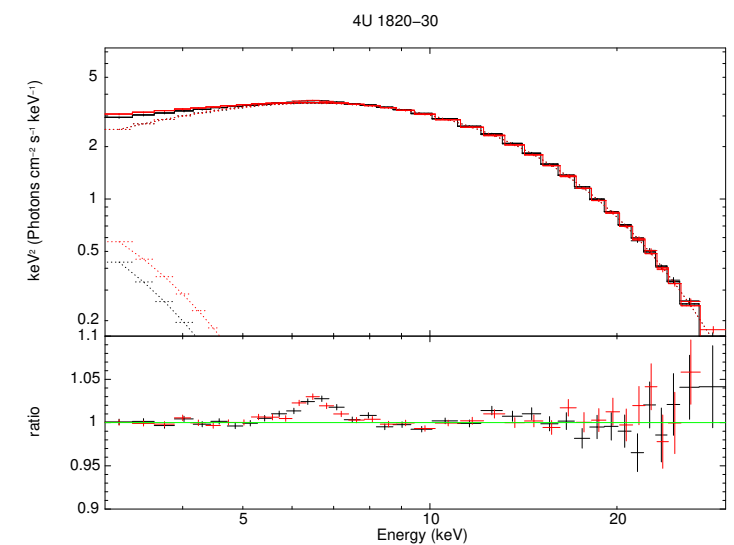

Figure A14. 4U 1820-30: Unfolded spectrum. Energy and data-vs-model ratio plot, for only the continuum model.

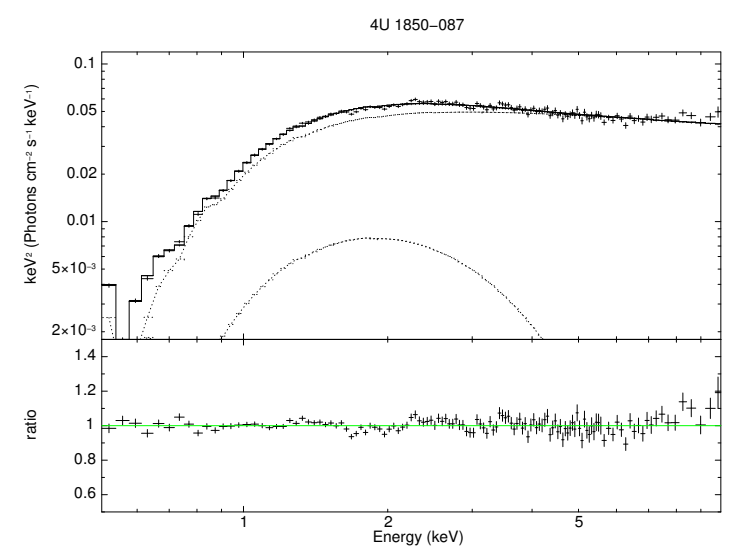

Figure A15. 4U 1850-087: Unfolded spectrum. Energy and data-vs-model ratio plot, for only the continuum model. 


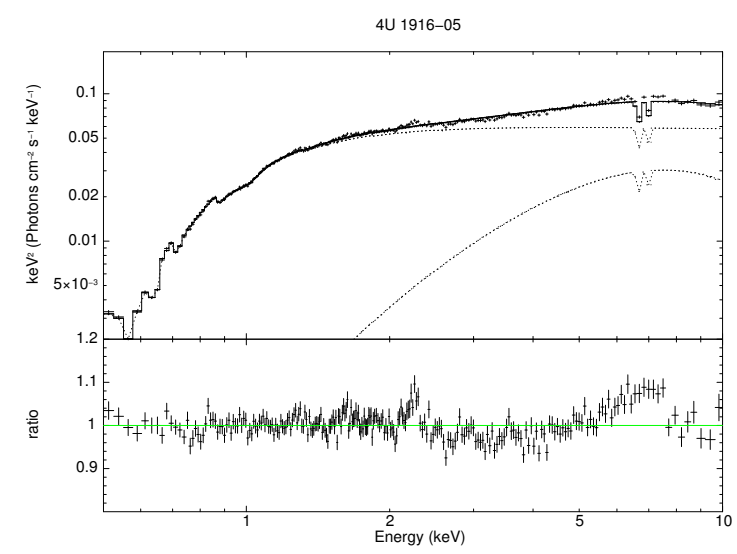

Figure A16. 4U 1916-05: Unfolded spectrum. Energy and data-vs-model ratio plot, for only the continuum model.

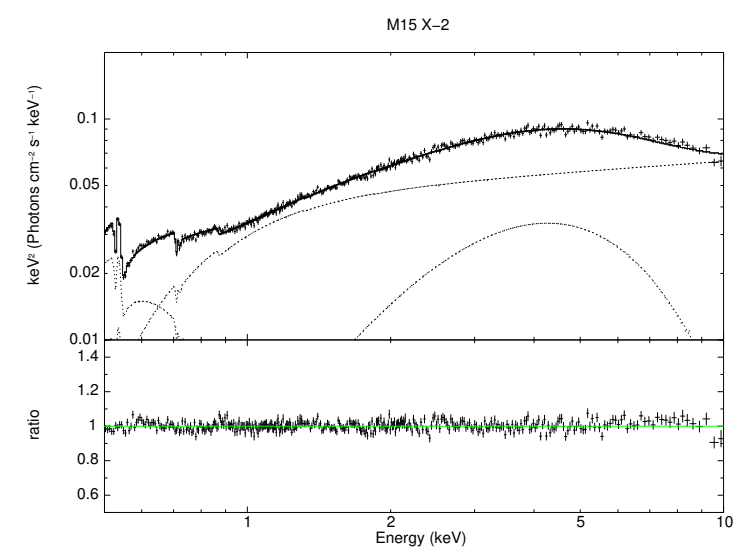

Figure A17. M15 X-2: Unfolded spectrum. Energy and data-vs-model ratio plot, for only the continuum model.

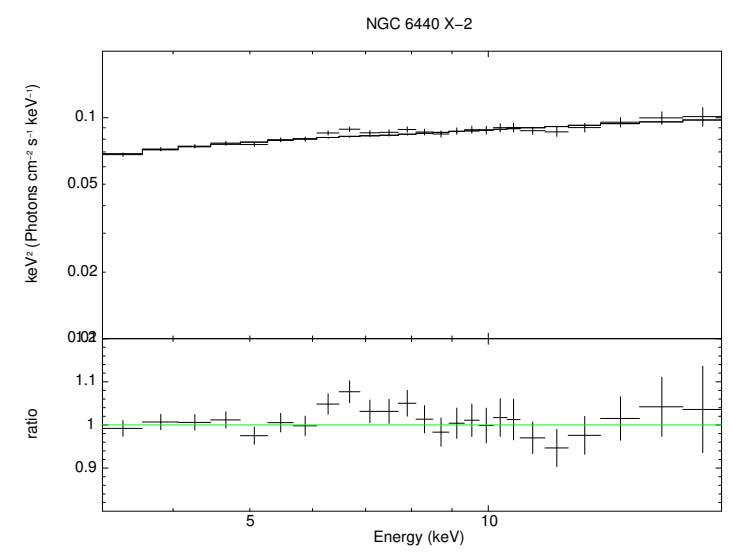

Figure A18. NGC 6440 X-2: Unfolded spectrum. Energy and data-vsmodel ratio plot, for only the continuum model. 